\title{
A Two-Level Game Theory Approach for Joint Relay Selection and Resource Allocation in Network Coding Assisted D2D Communications
}

\author{
Chuhan Gao, Yong Li, Senior Member, IEEE, Yulei Zhao, and Sheng Chen, Fellow, IEEE
}

\begin{abstract}
Device-to-device (D2D) communication, which enables direct transmissions between mobile devices to improve spectrum efficiency, is one of the preferable candidate technologies for the next generation cellular network. Network coding one the other hand is widely used to improve throughput in ad hoc networks. Thus, the performance of D2D communications in cellular networks can potentially benefit from network coding. Aiming to improve the achievable capacity of D2D communications, we propose a system with inter-session network coding enabled to assist D2D transmissions. We formulate the joint problem of relay selection and resource allocation in network coding assisted D2D communications, and obtain the overall capacity of the network under complex interference conditions as a function of the relay selection and resource allocation. To solve the formulated problem, we propose a two-level de-centralized approach, which solves the relay selection and resource allocation problems alternatively to obtain stable solutions for these two problems. Specifically, a coalition formation game and a greedy algorithm based game are utilized to solve these two problems, respectively. The performances of the proposed scheme is evaluated through extensive simulations to prove its superiority.
\end{abstract}

Index Terms-Device-to-device communication, network coding, relay selection, resource allocation, game theory

\section{INTRODUCTION}

Demand for mobile Internet access is growing at a tremendous rate. To satisfy this explosive traffic demand, deviceto-device (D2D) communication has been proposed for LongTerm Evaluation-Advanced [1]. In D2D communications, user equipments (UEs) in close proximity set up direct links for data transmissions, using licensed cellular spectrum resources, instead of through base stations (BSs). The benefits of such proximity communication is manifold [2]. It has the potential to provide extremely high bit rate as well as low end-to-end delay and power consumption due to short-range transmissions. Since the cellular resources can be simultaneously shared and utilized by D2D UEs, the spectrum efficiency and reuse gain are improved. In addition, D2D communications enable mobile

C. Gao, Y. Li and Y. Zhao (gch12@mails.tsinghua.edu.cn, liyong07@tsinghua.edu.cn,zyl@mails.tsinghua.edu.cn) are with Department of Electronic Engineering, Tsinghua University, Beijing 100084, China.

S. Chen (sqc@ecs.soton.ac.uk) is with Electronics and Computer Science, University of Southampton, Southampton SO17 1BJ, UK, and also with King Abdulaziz University, Jeddah 21589, Saudi Arabia.

This work was partially supported by the National Natural Science Foundation of China (NNSFC) under grants No. 61201189 and No. 61132002, the Creative Research Groups of NNSFC under grant No. 61021001, the National S\&T Major Project under grant No. 2011ZX03004-001-01, the National High Tech (863) Projects under Grant No. 2011AA010202, the Research Fund of Tsinghua University under grants No. 2011 Z05117 and No. 20121087985, and Tsinghua University Initiative Scientific Research Program. traffic offloading by user cooperations for content downloading and sharing, which also benefits cellular (non-D2D) users. Therefore, D2D communication is expected to be a key feature supported by the next-generation cellular network [3].

Although D2D communication enhances the system performance in many aspects, it also causes severe interference, which may degrade the transmission rates of both cellular and D2D users. To solve this problem, current works focus on resource allocation [1], [4]-[6] and power control [7], [8]. Gu et al. [1] utilized two stable matching algorithms to optimize the overall system throughput while simultaneously meeting the quality of service (QoS) requirements for cellular and D2D users. In the model of [1], D2D users seek channel reuse partners from cellular users to share their spectrum resources for data transmissions. Li et al. [4] considered a similar system model to solve the resource allocation problem by a coalition formation game based scheme. In the approach of [4], different transmission modes, mutual interferences and resource sharing policy are combined in a utility function, which is used by D2D users to determine the spectrum resource reuse partners in the coalition formation game. Yu et al. [5] optimized the throughput over the shared resources for D2D communications to improve local services, while fulfilling prioritized cellular service constraints. Xu et al. [6] introduced a reverse iterative combinatorial auction as the resource allocation mechanism to optimize the system sum rate. Lee et al. [7] proposed a random network model for D2D communications using stochastic geometry and developed centralized and distributed power control algorithms, while Liu et al. [8] analyzed the benefits of power control in enhancing the transmission capacity region.

Network coding has shown the potential to improve throughput efficiency [9], [10]. Current studies on network coding can be divided into two categories: intra-session coding [11], [12], and inter-session coding [13], [14]. Intra-session coding usually relies on random linear network coding to organize packets in groups to be linearly combined using randomly chosen coefficients from the elements of a finite field. Ho et al. [11] introduced dynamic algorithms for multicast routing, network coding, power allocation, session scheduling and rate allocation across correlated sources for intra-session network coding. Cai et al. [12] investigated the problem of selecting the candidate forwarder set and allocated traffic among candidate forwarders to achieve optimal routing in opportunistic routing with intra-session network coding. Since only packets from the same flow are combined in intra-session coding, it is 
not a good fit for D2D communications underlaying cellular networks, where each D2D pair is viewed as a flow, and the cooperation among different D2D pairs should be considered. Inter-session coding combines the packets from different network flows. Upon identifying sets of nodes that can form a coding region, the packets can be mixed (XORed) in order to attain higher spectrum efficiency. Katti et al. [13] proposed an architecture for wireless mesh networks, where routers mix packets from different sources to increase the information content of each transmission in addition to forwarding packets. Liu et al. [14] addressed the distributed control problem in heterogeneous-service networks with multi-rate multicast and unicast services, and proposed a decentralized rate control algorithm for inter-session network coding. Some recent works combined both intra-session coding and inter-session coding to enhance the system performance [15], [16].

Pahlevani et al. [10] discussed the potential of enabling network coding in D2D communications to enhance communication efficiency and security. In the scenario where a D2D community is composed of multiple devices connected in a multi-hop fashion, network coding's ability to recode coded packets on the fly provides the means of improving D2D communication's throughput, delay, and energy efficiency. A few schemes that enable network coding in D2D communications have been proposed so far, and they can be categorized into two types: either studying the relay selection problem [17], [18], or investigating the resource allocation problem [19], [20]. Specifically, Bhorkar et al. [17] investigated the relay selection and scheduling problems, while Maher et al. [18] utilized idle devices in D2D communications as relay nodes to enable network coding. Wu et al. [19] developed a radio resource management mechanism to optimize power control and subchannel allocation with network coding, while Wei et al. [20] investigated multi-pair D2D communications with a multi-antenna relay employing space-time analog network coding. However, in D2D communications underlaying cellular networks, both relay selection and resource allocation have major impact on the achievable performance of network coding and, moreover, these two problems are actually coupled and cannot be solved separately without compromising the overall system performance. Detailed discussions on the coupling of these two problems will be given later.

In this paper, we aim to assist the D2D communications underlaying cellular network with inter-session network coding. Specifically, we consider the scenario where relays assist nearby D2D pairs, forming coding regions, and perform network coding. In order to achieve high sum capacity, the problems of relay selection and resource allocation need to be solved. Firstly, the capacity gain highly depends on the locations of the relays and the assisted users. Therefore, D2D pairs need to select proper relays to assist their transmissions for maximizing the benefits of network coding. Secondly, the spectrum resources should also be allocated wisely to the D2D pairs and relays to mitigate the severe interference. We formulate a joint problem of relay selection and resource allocation, where these two problems are optimized jointly. Theoretically, the joint optimal solution can be obtained by exhaustive search. However, it is very difficult to apply any existing optimization approaches to solve this joint optimization efficiently owing to its extremely high computation complexity. Moreover, global network information, such as network topology, are required, which either impose high synchronizing overhead or are not available. Thus, we address these two problems from a game theory point of view, using a coalition formation game and a greedy algorithm based game. Utilizing game theory enables us to obtain the solutions for the relay selection and resource allocation problems efficiently, where the nodes only require local network information. More specifically, we propose a two-level optimization approach, termed NC-D2D, to obtain near-optimal solutions. In our NC-D2D, relay selection game and resource allocation game operate alternately, each using the results obtained by the other game as inputs, and the alternating optimization procedure continues until the system reaches a stable state in terms of both relay selection and resource allocation. Our contribution is three-fold, as summarized in the following.

- We introduce inter-session coding to assist D2D communications underlaying cellular networks with realistic multiple D2D pairs, relays, and cellular users, where D2D pairs select relays and form coding regions to improve the achievable capacity. Relays XOR the received packets before multicasting them to the corresponding D2D users.

- We formulate the joint problem of relay selection and resource allocation to maximize the sum transmission capacity of all D2D pairs and cellular users. The sum capacity of the network is derived as a function of the results of relay selection and resource allocation, while taking into account the interference among users.

- Since the joint optimal solution of relay selection and resource allocation imposes extremely high complexity, rendering it impractical for large-scale systems, we propose a two-level optimization approach, termed NC-D2D, to obtain stable solution for the joint optimization, where relay selection and resource allocation are performed alternately, utilizing a coalition formation game and a greedy algorithm based game, respectively. In each round, the input of one problem is provided by the solution of the other problem. In addition, each node only requires local information to solve the two problems. Through our extensive evaluation, we prove that our NC-D2D is able to obtain a stable near-optimal solution with very low computation complexity and in a few rounds.

The rest of the paper is organized as follows. After presenting the system model of network coding assisted D2D communications in Section 2, we derive the system capacity and formulate the joint problem of relay selection and resource allocation in Section 3. Then we give an overview of the proposed two-level NC-D2D optimization approach in Section 4, and detail the relay selection coalition formation game and resource allocation game in Sections 5 and 6, respectively. The performance of our proposed scheme is evaluated through extensive simulations in Section 7. Finally, we conclude this paper in Section 8. 


\section{System OVerview ANd Model}

\subsection{System Overview}

We focus on the scenario of a single cell involving multiple D2D pairs, relays and cellular users. In D2D communications, a pair of UEs in close proximity are able to enjoy extremely high data rate by setting up a direct link between them. However, it is well known that the channel quality between two users degrades rapidly as the distance between the transmitter and receiver increases. When the distance between the D2D transmitter and receiver is too long to support a direct link, these D2D users will have to switch to cellular mode [21], [22]. Therefore, we enable cooperative relays to assist D2D pairs for data transmissions in order to overcome the long distance between D2D users. Each D2D pair can either transmit data via the direct link between the two users, or assisted by a cooperative relay, depending on their circumstance. Hence there are two kinds of D2D pairs in the system, i.e., (i) ordinary D2D pair: two D2D users transmit via the direct link between them, and (ii) relay Assisted D2D pair: two D2D users are assisted by a relay which employs network coding aided transmission. We denote the sets of all D2D pairs, relays and cellular users as $\mathcal{D}, \mathcal{R}$ and $\mathcal{U}$, respectively. D2D pair $i$ is denoted by $d_{i}$, while its transmitter and receiver is denoted by $d_{i}^{t}$ and $d_{i}^{r}$. Relay $i$ and cellular user $j$ is denoted as $r_{i}$ and $u_{j}$, respectively. We allow multiple D2D pairs and relays to share the spectrum resource of one cellular user in order to increase the possible number of concurrent transmissions. To fully exploit this spatial reuse gain, therefore, the interference caused by this spectrum sharing must be taken into consideration.

As illustrated in Fig. 1, D2D pairs $d_{1}$ and $d_{2}$ are relay assisted D2D pairs, aided by relay $r_{1}$, while $d_{3}$ and $d_{4}$ are ordinary D2D pairs. $d_{1}$ and $d_{2}$ occupy the uplink spectrum resource of cellular user $u_{1}$ and $u_{2}$, respectively, and $u_{3}$ shares its resource with $r_{1}, d_{3}$ and $d_{4}$. The interferences between $d_{2}$ and $u_{2}$ as well as between $r$ and $d_{3}$ are plotted as examples.

We introduce network coding to aid D2D communications, which utilizes the relay to improve throughput efficiency. Due to the spectrum sharing, mutual interferences exist among D2D pairs, regular cellular users and cooperative relays. In order to achieve high transmission rates for D2D users and cellular users, both the relay selection for network coding and the spectrum resource allocation need to be optimized. In our system, the D2D pairs can choose different relays to assist their transmissions by applying network coding. However, the throughput gain highly depends on the distances and channel qualities of the links among the D2D users and relays that form a coding region, as will be analyzed in detail later. Moreover, the relays that are available in the network may not be enough to assist all the D2D pairs. In this case, they should assist the D2D pairs that have long link distance or poor channel quality to maximize the throughput gain of network coding. Therefore, the D2D pairs should select proper working modes, namely, whether to rely on relays and to select which relays in order to achieve the maximum throughput gain. The limited spectrum resources shared by the cellular users should also be allocated properly to the D2D pairs and relays to ensure the QoS of all users and to achieve high sum rates of the network.

\subsection{System Model}

We define the binary variables $x_{d, u}, x_{r, u}$ and $y_{d, r}$ to depict the relay selection and resource allocation policies for D2D pairs and relays. Specifically, $x_{d, u}=1$ indicates that D2D pair $d$ uses the uplink resource of cellular user $u$, otherwise $x_{d, u}=0$, while $x_{r, u}=1$ if relay $r$ shares the spectrum resource of $u$, otherwise $x_{r, u}=0$. Similarly, $y_{d, r}=1$ indicates that relay $r$ assists the D2D pair $d$ 's transmission, otherwise, $y_{d, r}=0$. We denote the matrices of $x_{d, u}, x_{r, u}$ and $y_{d, r}$ as $\boldsymbol{X}_{\mathcal{D}}, \boldsymbol{X}_{\mathcal{R}}$ and $\boldsymbol{Y}$, respectively. Each row in $\boldsymbol{X}_{\mathcal{D}}$ and $\boldsymbol{X}_{\mathcal{R}}$ represents the spectrum resource sharing of the corresponding D2D pair and relay, respectively, while each row in $\boldsymbol{Y}$ represents the relay selection of the corresponding D2D pair. For example, in the scenario of Fig. 1, these three matrices can be written as:

$$
\begin{aligned}
\boldsymbol{X}_{\mathcal{D}} & =\left[\begin{array}{lll}
1 & 0 & 0 \\
0 & 1 & 0 \\
0 & 0 & 1 \\
0 & 0 & 1
\end{array}\right], \\
\boldsymbol{X}_{\mathcal{R}} & =\left[\begin{array}{lll}
0 & 0 & 1
\end{array}\right], \\
\boldsymbol{Y} & =\left[\begin{array}{llll}
1 & 1 & 0 & 0
\end{array}\right]^{T} .
\end{aligned}
$$

\subsection{Network Coding Assisted D2D Transmissions}

We adopt an inter-session network coding to assist the transmissions of D2D pairs. A typical coding region is presented in Fig. 2, which consists of two relay assisted D2D pairs and one relay. This scheme is described in [10], [16]. We will first describe the operation of this scheme in our system. Then we will derive the achievable rate of this coding region, and analyze the capacity gain of network coding in Section 3.

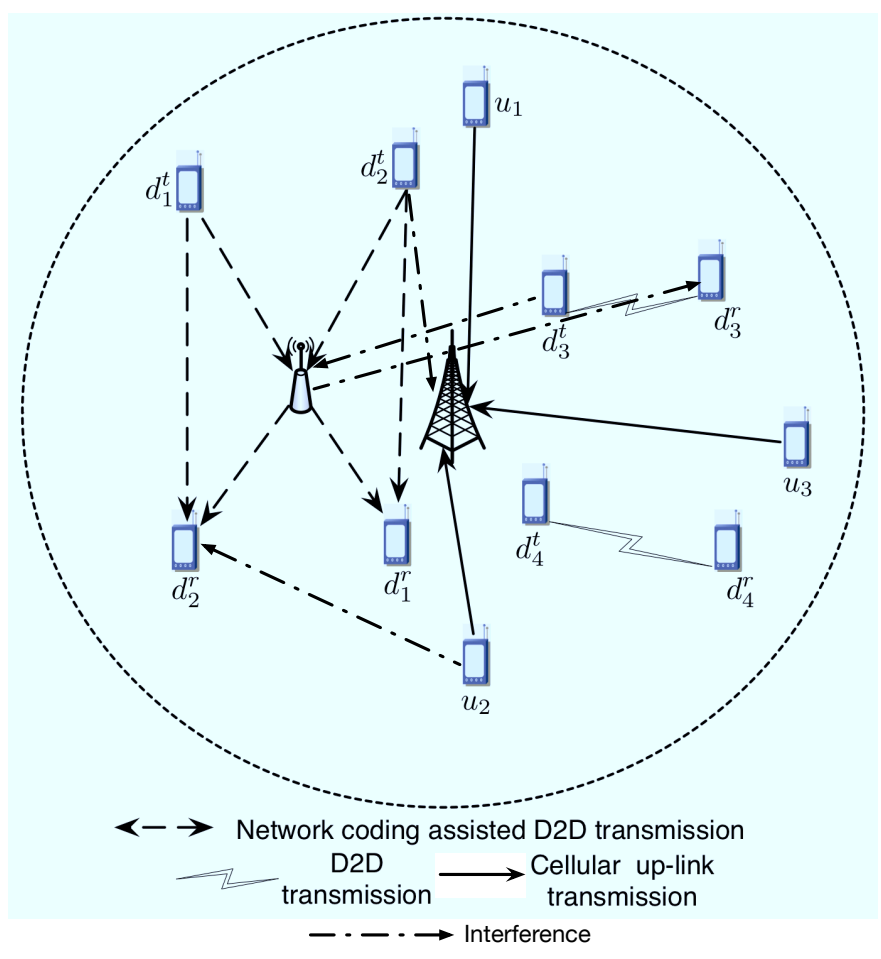

Fig. 1. Illustration of the network coding aided D2D communications underlaying cellular network, where there are 3 cellular users, 4 D2D pairs and 1 cooperative relay. 


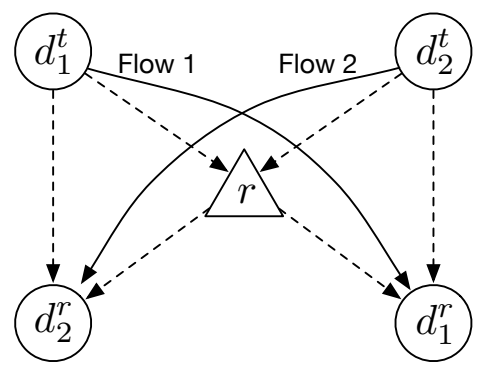

Fig. 2. Illustration of a coding region in network coding assisted D2D communications.

For an ordinary D2D pair, the data is transmitted via the direct link between the two devices. For the relay assisted D2D pairs as shown in Fig. 2, sender $d_{1}^{t}$ multicasts the packets to relay $r$ and $d_{2}^{r}$, while $d_{2}^{t}$ multicasts the packets to $r$ and $d_{1}^{r}$. Relay $r$ XORs the packets received from $d_{1}^{t}$ and $d_{2}^{t}$ bit by bit before multicasting them to $d_{1}^{r}$ and $d_{2}^{r}$. Then $d_{1}^{r}$ and $d_{2}^{r}$ are able to decode the packets after receiving the XORed packets from $r$ and the multicasted packets from $d_{2}^{t}$ and $d_{1}^{t}$, respectively. The assist of relay by applying network coding not only helps to overcome the low transmission rate of the direct link between users, caused by long transmitting distance or poor channel quality, but also improves the spectrum efficiency.

\section{Problem Formulation}

We first derive the achievable capacity of network coding assisted D2D communications and compare it with that of ordinary D2D communications without the assist of network coding and cooperative relays, based on which we then obtain the sum capacity of the network as a function of $\boldsymbol{X}_{\mathcal{D}}, \boldsymbol{X}_{\mathcal{R}}$ and $\boldsymbol{Y}$. Finally, we formulate the optimization problem of relay selection and resource allocation with system constrains.

\subsection{Network Coding Assisted D2D Capacity}

We first consider one coding region, which consists of one relay and two relay assisted D2D pairs as shown in Fig. 2, and derive its capacity. Assume that $d_{1}^{t}$ wishes to send packet $A$ to $d_{1}^{r}$ and $d_{2}^{t}$ wishes to send packet $B$ to $d_{2}^{r}$. If $d_{1}$ and $d_{2}$ work under ordinary mode, i.e., there is no relay to assist them, $d_{1}^{t}$ and $d_{2}^{t}$ will transmit $A$ and $B$ via links $\left(d_{1}^{t}, d_{1}^{r}\right)$ and $\left(d_{2}^{t}, d_{2}^{r}\right)$, respectively. Therefore, the achievable capacity of these two D2D pairs is $c\left(d_{1}^{t}, d_{1}^{r}\right)+c\left(d_{2}^{t}, d_{2}^{r}\right)$, where $c(i, j)$ represents the capacity of link $(i, j)$. With the aid of network coding, $d_{1}^{t}$ multicasts $A$ to $d_{2}^{r}$ and $r$, and $d_{2}^{t}$ multicasts $B$ to $d_{1}^{r}$ and $r$. Then $r$ combines $A$ and $B$, and multicasts $A$ XOR $B$ to $d_{1}^{r}$ and $d_{2}^{r}$, who will be able to decode $A$ and $B$, respectively.

Since relay $r$ needs to combine the packets from both $d_{1}^{t}$ and $d_{2}^{t}$ and then multicasts the result, the rate that $r$ sends $A$ XOR $B$ to $d_{1}^{r}$ and $d_{2}^{r}$ is actually limited by the transmitting rates of the four links: $\left(d_{1}^{t}, r\right),\left(d_{2}^{t}, r\right),\left(r, d_{1}^{r}\right)$ and $\left(r, d_{2}^{r}\right)$. Firstly, the maximum rate that $r$ is able to send $A$ XOR $B$ is limited by the lower-rate link of the two, $\left(d_{1}^{t}, r\right)$ and $\left(d_{2}^{t}, r\right)$, which has the rate $\min \left\{c\left(d_{1}^{t}, r\right), c\left(d_{2}^{t}, r\right)\right\}$. The computing time of $r$ to combine $A$ and $B$ is neglected compared to packets' transmission time. Then $A$ XOR $B$ is transmitted through one more hop, link $\left(r, d_{1}^{r}\right)$ to $d_{1}^{r}$. For the purpose of deriving the achievable rate, the process of $d_{1}^{r}$ receiving $A$ XOR $B$ can be modeled as a two-hop transmission, where $r$ receives $A$ XOR $B$ from a virtual source node and then transmits it to $d_{1}^{r}$. The transmitting rate of the second hop is obviously $c\left(r, d_{1}^{r}\right)$, and the transmitting rate of the first virtual hop is equivalent to the rate of $r$ receiving $A$ and $B$, which is $\min \left\{c\left(d_{1}^{t}, r\right), c\left(d_{2}^{t}, r\right)\right\}$. For this two-hop transmission, therefore, the achievable rate is equal to $\min \left\{c\left(r, d_{1}^{r}\right), \min \left\{c\left(d_{1}^{t}, r\right), c\left(d_{2}^{t}, r\right)\right\}\right\}$. In addition to $A$ XOR $B, d_{1}^{r}$ also needs to receive packet $B$ from $d_{2}^{t}$ in order to decode $A$ from $A$ XOR $B$. The rate of $d_{1}^{r}$ receiving $B$ is $c\left(d_{2}^{t}, d_{1}^{r}\right)$. Thus, the rate of $d_{1}^{r}$ receiving (decoding) $A$ is $\min \left\{c\left(d_{2}^{t}, d_{1}^{r}\right), \min \left\{c\left(r, d_{1}^{r}\right), \min \left\{c\left(d_{1}^{t}, r\right), c\left(d_{2}^{t}, r\right)\right\}\right\}\right\}$. Similarly, the rate $d_{2}^{r}$ receiving (decoding) $B$ can be shown to be $\min \left\{c\left(d_{1}^{t}, d_{2}^{r}\right), \min \left\{c\left(r, d_{2}^{r}\right), \min \left\{c\left(d_{1}^{t}, r\right), c\left(d_{2}^{t}, r\right)\right\}\right\}\right\}$. The total achievable capacity of the coding region formed by relay $r$ and D2D pairs $d_{1}$ and $d_{2}$ is therefore given by

$$
\begin{aligned}
& c_{\mathcal{C} \mathcal{R}}\left(r, d_{1}, d_{2}\right)= \\
& \min \left\{c\left(d_{2}^{t}, d_{1}^{r}\right), \min \left\{c\left(r, d_{1}^{r}\right), \min \left\{c\left(d_{1}^{t}, r\right), c\left(d_{2}^{t}, r\right)\right\}\right\}\right\}+ \\
& \min \left\{c\left(d_{1}^{t}, d_{2}^{r}\right), \min \left\{c\left(r, d_{2}^{r}\right), \min \left\{c\left(d_{1}^{t}, r\right), c\left(d_{2}^{t}, r\right)\right\}\right\}\right\},
\end{aligned}
$$

which can be simplified to

$$
\begin{aligned}
& c_{\mathcal{C} \mathcal{R}}\left(r, d_{1}, d_{2}\right)=\min \left\{c\left(d_{2}^{t}, d_{1}^{r}\right), c\left(r, d_{1}^{r}\right), c\left(d_{1}^{t}, r\right), c\left(d_{2}^{t}, r\right)\right\} \\
& \quad+\min \left\{c\left(d_{1}^{t}, d_{2}^{r}\right), c\left(r, d_{2}^{r}\right), c\left(d_{1}^{t}, r\right), c\left(d_{2}^{t}, r\right)\right\} .
\end{aligned}
$$

With the achievable capacity of network coding assisted D2D communications in (5), we also need the capacity of each link in order to derive the system capacity as a function of $\boldsymbol{X}_{\mathcal{D}}, \boldsymbol{X}_{\mathcal{R}}$ and $\boldsymbol{Y}$. The transmission capacity of a link is

$$
c=\log _{2}\left(\frac{P_{R}}{I+N}+1\right),
$$

where $P_{R}$ is the receiving power at the receiver of the link, while $I$ and $N$ denote the interference and noise powers, respectively. We assume Rayleigh fading and adopt the Friis transmission equation to calculate the path loss of the transmitted signal [23]. Therefore, $P_{R}$ can be written as

$$
P_{R}=P_{T}+G_{T}+G_{R}+20 \lg \left(\frac{\lambda}{4 \pi l}\right)
$$

where $P_{T}$ is the transmitting power, $G_{T}$ and $G_{R}$ are the antenna gains of the transmitter and receiver, respectively, while $\lambda$ is the signal wavelength and $l$ is the link distance. The transmitting powers of relay, D2D user and cellular user are labeled as $P_{T}^{\mathcal{R}}, P_{T}^{\mathcal{D}}$ and $P_{T}^{\mathcal{U}}$, respectively. Similarly, we have $G_{T}^{\mathcal{R}}, G_{T}^{\mathcal{D}}, G_{T}^{\mathcal{U}}, G_{R}^{\mathcal{R}}, G_{R}^{\mathcal{D}}$ and $G_{R}^{\mathcal{U}}$ for these three kinds of users, and $G_{R}^{\mathcal{B S}}$ for the BS. For simplicity, we denote $\widetilde{P}_{T}=P_{T}+G_{T}+G_{R}$ in the sequel.

For the purpose of relay selection and resource allocation as well as deriving the system capacity, we need to know which nodes form coding regions with a given matrix $\boldsymbol{Y}$. In other words, given a relay $r_{j}$, we want to represent the two D2D pairs it assisted. We label the D2D pairs assisted by $r_{j}$ as $\alpha(j)$ and $\beta(j)$. According to the definition of $\boldsymbol{Y}, y_{d_{\alpha(j)}, r_{j}}=$ $y_{d_{\beta(j)}, r_{j}}=1$, while $y_{d_{i}, r_{j}}=0 \forall i \neq \alpha(j), \beta(j)$. Therefore, we have the following equation set:

$$
\left\{\begin{array}{l}
\alpha(j)+\beta(j)=\sum_{i} y_{d_{i}, r_{j}} \cdot i=a, \\
\alpha(j)^{2}+\beta(j)^{2}=\sum_{i} y_{d_{i}, r_{j}} \cdot i^{2}=b .
\end{array}\right.
$$


Solving this equation set yields

$$
\left\{\begin{array}{l}
\alpha(j)=\frac{a+\sqrt{2 b-a^{2}}}{2}, \\
\beta(j)=\frac{a-\sqrt{2 b-a^{2}}}{2},
\end{array}\right.
$$

which give the D2D pairs $d_{\alpha(j)}$ and $d_{\beta(j)}$ assisted by relay $r_{j}$.

To obtain the capacity of each link, we need considering the interference. There are three types of links involving D2D users in each coding region: the links from D2D transmitters to D2D receivers (links $\left(d_{1}^{t}, d_{2}^{r}\right)$ and $\left(d_{2}^{t}, d_{1}^{r}\right)$ in Fig. 2), the links from D2D transmitters to the relay (links $\left(d_{1}^{t}, r\right)$ and $\left(d_{2}^{t}, r\right)$ ), and the links from relay to D2D receivers (links $\left(r, d_{1}^{r}\right)$ and $\left.\left(r, d_{2}^{r}\right)\right)$. Thus, the interference can be categorized into three kinds: the interference from other D2D users, interference from relays, and the interference from cellular users.

For the coding region of relay $r_{j}$, first consider $\left(d_{\alpha(j)}^{t}, d_{\beta(j)}^{r}\right)$ and $\left(d_{\beta(j)}^{t}, d_{\alpha(j)}^{r}\right)$. For $\left(d_{\alpha(j)}^{t}, d_{\beta(j)}^{r}\right)$, the interference from other D2D transmitters, denoted by $I_{\left(d_{\alpha(j)}^{t}, d_{\beta(j)}^{r}\right)}^{\mathcal{D}}$, is

$$
\begin{aligned}
I_{\left(d_{\alpha(j)}^{t}, d_{\beta(j)}^{r}\right)}^{\mathcal{D}}= & \sum_{m} \sum_{k \neq \alpha(j)} x_{d_{\alpha(j)}, u_{m}} \\
& \cdot x_{d_{k}, u_{m}}\left(\widetilde{P}_{T}+20 \lg \left(\frac{\lambda}{4 \pi l_{\left(d_{k}^{t}, d_{\beta(j)}^{r}\right)}}\right)\right),
\end{aligned}
$$

while the interference from relays, denoted by $I_{\left(d_{\alpha(j)}^{t}, d_{\beta(j)}^{r}\right)}^{\mathcal{R}}$, can be expressed as

$$
\begin{aligned}
I_{\left(d_{\alpha(j)}^{t}, d_{\beta(j)}^{r}\right)}^{\mathcal{R}}= & \sum_{m} \sum_{k} x_{d_{\alpha(j)}, u_{m}} \\
& \cdot x_{r_{k}, u_{m}}\left(\widetilde{P}_{T}+20 \lg \left(\frac{\lambda}{4 \pi l_{\left(r_{k}, d_{\beta(j)}^{r}\right)}}\right)\right) .
\end{aligned}
$$

Similarly, the interference from cellular users, denoted by $I_{\left(d_{\alpha(j)}^{t}, d_{\beta(j)}^{r}\right)}^{\mathcal{U}}$, can be expressed as,

$$
I_{\left(d_{\alpha(j)}^{t}, d_{\beta(j)}^{r}\right)}^{\mathcal{U}}=\sum_{m} x_{d_{\alpha(j)}, u_{m}}\left(\widetilde{P}_{T}+20 \lg \left(\frac{\lambda}{4 \pi l_{\left(u_{m}, d_{\beta(j)}^{r}\right)}}\right)\right) .
$$

Therefore, we have

$$
\begin{aligned}
& c\left(d_{\alpha(j)}^{t}, d_{\beta(j)}^{r}\right)= \\
& \log _{2}\left(\frac{\widetilde{P}_{T}+20 \lg \left(\frac{\lambda}{4 \pi l_{\left(d_{\alpha(j)}^{t}, d_{\beta(j)}^{r}\right)}}\right)}{I_{\left(d_{\alpha(j)}^{\mathcal{D}}, d_{\beta(j)}^{r}\right)}^{\mathcal{D}}+I_{\left(d_{\alpha(j)}^{t}, d_{\beta(j)}^{r}\right)}^{\mathcal{R}}+I_{\left(d_{\alpha(j)}^{t}, d_{\beta(j)}^{r}\right)}^{\mathcal{U}}+N}+1\right) .
\end{aligned}
$$

In the same way, the capacity of link $\left(d_{\beta(j)}^{t}, d_{\alpha(j)}^{r}\right)$ is

$$
\begin{aligned}
& c\left(d_{\beta(j)}^{t}, d_{\alpha(j)}^{r}\right)= \\
& \log _{2}\left(\frac{\widetilde{P}_{T}+20 \lg \left(\frac{\lambda}{4 \pi l_{\left(d_{\beta(j)}^{t}, d_{\alpha(j)}^{r}\right)}}\right)}{I_{\left(d_{\beta(j)}^{t}, d_{\alpha(j)}^{r}\right)}^{d}+I_{\left(d_{\beta(j)}^{t}, d_{\alpha(j)}^{r}\right)}^{r}+I_{\left(d_{\beta(j)}^{t}, d_{\alpha(j)}^{r}\right)}^{u}+N}+1\right) .
\end{aligned}
$$

Next consider $\left(d_{\alpha(j)}^{t}, r_{j}\right)$ and $\left(d_{\beta(j)}^{t}, r_{j}\right)$. For $\left(d_{\alpha(j)}^{t}, r_{j}\right)$, the interferences from other D2D users, relays and cellular users can be expressed respectively as

$$
\begin{aligned}
& I_{\left(d_{\alpha(j)}^{t}, r_{j}\right)}^{\mathcal{D}}= \sum_{m} \sum_{k \neq i} x_{d_{\alpha(j)}, u_{m}} \\
& \cdot x_{d_{k}, u_{m}}\left(\widetilde{P}_{T}+20 \lg \left(\frac{\lambda}{4 \pi l_{\left(d_{k}^{t}, r_{j}\right)}}\right)\right), \\
& I_{\left(d_{\alpha(j)}^{t}, r_{j}\right)}^{\mathcal{R}}= \sum_{m} \sum_{k \neq j} x_{d_{\alpha(j)}, u_{m}} \\
& \cdot x_{r_{k}, u_{m}}\left(\widetilde{P}_{T}+20 \lg \left(\frac{\lambda}{4 \pi l_{\left(r_{k}, r_{j}\right)}}\right)\right), \\
& I_{\left(d_{\alpha(j)}^{t}, r_{j}\right)}^{\mathcal{U}}=\sum_{m} x_{d_{\alpha(j)}, u_{m}}\left(\widetilde{P}_{T}+20 \lg \left(\frac{\lambda}{4 \pi l_{\left(u_{m}, r_{j}\right)}}\right)\right) .
\end{aligned}
$$

Thus we have

$$
\begin{aligned}
& c\left(d_{\alpha(j)}^{t}, r_{j}\right)= \\
& \log _{2}\left(\frac{\widetilde{P_{T}}+20 \lg \left(\frac{\lambda}{4 \pi l_{\left(d_{\alpha(j)}^{t}, r_{j}\right)}}\right)}{I_{\left(d_{\alpha(j)}^{t}, r_{j}\right)}^{\mathcal{D}}+I_{\left(d_{\alpha(j)}^{t}, r_{j}\right)}^{\mathcal{R}}+I_{\left(d_{\alpha(j)}^{t}, r_{j}\right)}^{\mathcal{U}}+N}+1\right), \\
& c\left(d_{\beta(j)}^{t}, r_{j}\right)= \\
& \log _{2}\left(\frac{\widetilde{P}_{T}+20 \lg \left(\frac{\lambda}{4 \pi l_{\left(d_{\beta(j)}^{t}, r_{j}\right)}}\right)}{I_{\left(d_{\beta(j)}^{t}, r_{j}\right)}^{\mathcal{D}}+I_{\left(d_{\beta(j)}^{t}, r_{j}\right)}^{\mathcal{R}}+I_{\left(d_{\beta(j)}^{t}, r_{j}\right)}^{\mathcal{U}}+N}+1\right) .
\end{aligned}
$$

Finally, consider $\left(r_{j}, d_{\alpha(j)}^{r}\right)$ and $\left(r_{j}, d_{\beta(j)}^{r}\right)$. For $\left(r_{j}, d_{\alpha(j)}^{r}\right)$, the three types of interference can be written respectively as

$$
\begin{aligned}
I_{\left(r_{j}, d_{\alpha(j)}^{r}\right)}^{\mathcal{D}}= & \sum_{m} \sum_{k} x_{r_{j}, u_{m}} \\
& \cdot x_{d_{k}, u_{m}}\left(\widetilde{P}_{T}+20 \lg \left(\frac{\lambda}{4 \pi l_{\left(d_{k}^{t}, d_{\alpha(j)}^{r}\right)}}\right)\right) \\
I_{\left(r_{j}, d_{\alpha(j)}^{r}\right)}^{\mathcal{R}}= & \sum_{m} \sum_{k \neq j} x_{r_{j}, u_{m}} \\
& \cdot x_{r_{k}, u_{m}}\left(\widetilde{P}_{T}+20 \lg \left(\frac{\lambda}{\left.4 \pi l_{\left(r_{k}, d_{\alpha(j)}^{r}\right)}\right)}\right)\right. \\
I_{\left(r_{j}, d_{\alpha(j)}^{r}\right)}^{\mathcal{U}}= & \sum_{m} x_{d_{\alpha(j)}, u_{m}}\left(\widetilde{P}_{T}+20 \lg \left(\frac{\lambda}{\left.4 \pi l_{\left(u_{m}, d_{\alpha(j)}^{r}\right)}\right)}\right)\right) .
\end{aligned}
$$

Thus the link capacities can be expressed as

$$
\begin{aligned}
& c\left(r_{j}, d_{\alpha(j)}^{r}\right)= \\
& \log _{2}\left(\frac{\widetilde{P}_{T}+20 \lg \left(\frac{\lambda}{4 \pi l_{\left(r_{j}, d_{\alpha(j)}^{r}\right)}}\right)}{I_{\left(r_{j}, d_{\alpha(j)}^{r}\right)}^{\mathcal{D}}+I_{\left(r_{j}, d_{\alpha(j)}^{r}\right)}^{\mathcal{R}}+I_{\left(r_{j}, d_{\alpha(j)}^{r}\right)}^{\mathcal{U}}+N}+1\right), \\
& c\left(r_{j}, d_{\beta(j)}^{r}\right)= \\
& \log _{2}\left(\frac{\widetilde{P}_{T}+20 \lg \left(\frac{\lambda}{4 \pi l_{\left(r_{j}, d_{\beta(j)}^{r}\right)}^{r}}\right)}{I_{\left(r_{j}, d_{\beta(j)}^{r}\right)}^{\mathcal{D}}+I_{\left(r_{j}, d_{\beta(j)}^{r}\right)}^{\mathcal{R}}+I_{\left(r_{j}, d_{\beta(j)}^{r}\right)}^{\mathcal{U}}+N}+1\right) .
\end{aligned}
$$

From (5), the total capacity of the coding region of relay $r_{j}$ can be rewritten as

$$
\begin{gathered}
c_{\mathcal{C R}}\left(r_{j}, d_{\alpha(j)}, d_{\beta(j)}\right)=\min \left\{c\left(d_{\beta(j)}^{t}, d_{\alpha(j)}^{r}\right), c\left(r_{j}, d_{\alpha(j)}^{r}\right),\right. \\
\left.c\left(d_{\alpha(j)}^{t}, r_{j}\right), c\left(d_{\beta(j)}^{t}, r_{j}\right)\right\}+\min \left\{c\left(d_{\alpha(j)}^{t}, d_{\beta(j)}^{r}\right)\right. \\
\left.c\left(r_{j}, d_{\beta(j)}^{r}\right), c\left(d_{\alpha(j)}^{t}, r_{j}\right), c\left(d_{\beta(j)}^{t}, r_{j}\right)\right\}
\end{gathered}
$$


in which all the link capacities can be calculated according to (13), (14), (18), (19), (23) and (24). It is possible that not all the relays form coding regions with D2D pairs and assist their transmissions. For any relay $r_{j}, \sum_{i} y_{d_{i}, r_{j}}=2$ if $r_{j}$ assists the transmissions of two D2D pairs, otherwise $\sum_{i} y_{d_{i}, r_{j}}=0$. Therefore, the sum capacity of all the relay assisted D2D pairs in the whole network, labeled as $c_{\mathcal{R} \mathcal{A}}$, can be expressed as

$$
c_{\mathcal{R} \mathcal{A}}=\frac{1}{2} \sum_{j} \sum_{i} y_{d_{i}, r_{j}} c_{\mathcal{C} \mathcal{R}}\left(r_{j}, d_{\alpha(j)}, d_{\beta(j)}\right) \text {. }
$$

\subsection{Network Coding Gain}

To compare the achievable capacities of D2D communications with and without relay assisted network coding, we define the network coding gain $G_{N C}$ as the increased sum capacity by applying relay aided network coding dividing by the sum capacity achieved without applying network coding. For the scenario in Fig. 2, $G_{N C}$ can be expressed as

$$
G_{N C}=\frac{c_{\mathcal{C R}}\left(r, d_{1}, d_{2}\right)-c\left(d_{1}^{t}, d_{1}^{r}\right)-c\left(d_{2}^{t}, d_{2}^{r}\right)}{c\left(d_{1}^{t}, d_{1}^{r}\right)+c\left(d_{2}^{t}, d_{2}^{r}\right)} .
$$

With the above definition, $G_{N C}=0$ means that the capacities with and without network coding are the same, while $G_{N C}=$ 1 means that network coding doubles the capacity.

To investigate the benefits of network coding, we evaluate this metric in the typical scenario of Fig. 2. The coordinates of the four D2D users in the network are as follows

$$
d_{1}^{t}(0,20), d_{1}^{r}(30,0), d_{2}^{t}(30,20), d_{2}^{r}(0,0) .
$$

We deploy relay $r$ in different positions. Specifically, $r$ 's $x$ coordinate varies from 1 to 29 , while $r$ 's $y$-coordinate varies from 1 to 19 . The setup of the simulation is explained in Section 7, and the simulation parameters can be found in Table 1. The achieved network coding gains $G_{N C}$ for these $29 \times 19$ network topologies are calculated and plotted in Fig. 3. As expected, the gain is highly related to the position of relay. Specifically, the capacity of relay assisted network coding increases as relay moves to the center, where the maximum system capacity is more than doubling that of simply transmitting via direct links between D2D users. $G_{N C}$ drops to around 0.1 when relay is located near the four D2D users, which corresponds to the four corners in the figure.

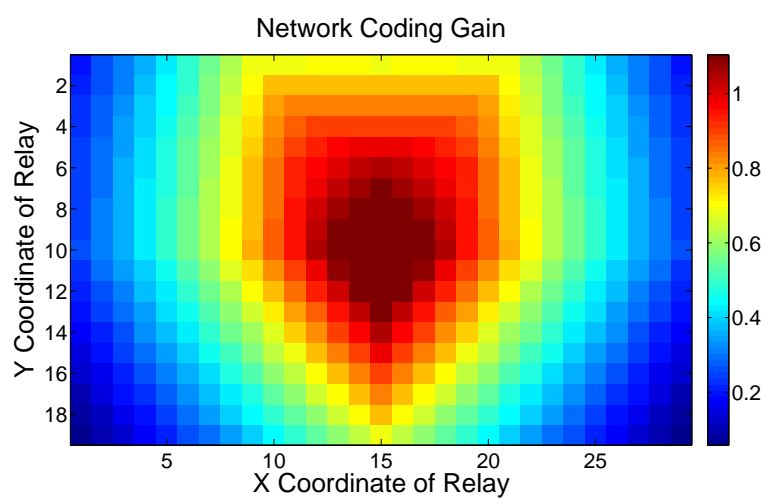

Fig. 3. Network coding gain $G_{N C}$ with relay deployed in different locations.

Based on the above results we can derive the conclusion that relay assisted network coding is capable of enhancing the system performance significantly. However, such benefits highly depend on the network topology. In particular, the capacity gain of applying network coding relies on selecting proper relays to assist the D2D pairs.

\subsection{Overall System Capacity}

To obtain the network sum capacity as a function of $\boldsymbol{X}_{\mathcal{D}}, \boldsymbol{X}_{\mathcal{R}}$ and $\boldsymbol{Y}$, we also need the sum capacities of ordinary D2D pairs and cellular users. For an ordinary D2D pair $d_{i}$ transmitting via link $\left(d_{i}^{t}, d_{i}^{r}\right)$, the interference from other D2D transmitters, relays and cellular users can be expressed respectively as

$$
\begin{aligned}
I_{\left(d_{i}^{t}, d_{i}^{r}\right)}^{\mathcal{D}}= & \sum_{m} \sum_{k \neq i} x_{d_{i}, u_{m}} \\
& \cdot x_{d_{k}, u_{m}}\left(\widetilde{P}_{T}+20 \lg \left(\frac{\lambda}{4 \pi l_{\left(d_{k}^{t}, d_{i}^{r}\right)}}\right)\right), \\
I_{\left(d_{i}^{t}, d_{i}^{r}\right)}^{\mathcal{R}}= & \sum_{m} \sum_{k} x_{d_{i}, u_{m}} \\
& \cdot x_{r_{k}, u_{m}}\left(\widetilde{P}_{T}+20 \lg \left(\frac{\lambda}{4 \pi l_{\left(r_{k}, d_{i}^{r}\right)}}\right)\right), \\
I_{\left(d_{i}^{t}, d_{i}^{r}\right)}^{\mathcal{U}}= & \sum_{m} x_{d_{i}, u_{m}}\left(\widetilde{P}_{T}+20 \lg \left(\frac{\lambda}{4 \pi l_{\left(u_{m}, d_{i}^{r}\right)}}\right)\right) .
\end{aligned}
$$

Thus the link capacity is

$$
c\left(d_{i}^{t}, d_{i}^{r}\right)=\log _{2}\left(\frac{\widetilde{P}_{T}+20 \lg \left(\frac{\lambda}{4 \pi l_{\left(d_{i}^{t}, d_{i}^{r}\right)}}\right)}{I_{\left(d_{i}^{t}, d_{i}^{r}\right)}^{\mathcal{D}}+I_{\left(d_{i}^{t}, d_{i}^{r}\right)}^{\mathcal{R}}+I_{\left(d_{i}^{t}, d_{i}^{r}\right)}^{\mathcal{U}}+N}+1\right) .
$$

For ordinary D2D pair $d_{i}, \sum_{j} y_{d_{i}, r_{j}}=0$. For relay assisted D2D pair $d_{k}, \sum_{j} y_{d_{k}, r_{j}}=1$. Therefore, the sum capacity of all ordinary D2D pairs in the system can be expressed as,

$$
c_{\mathcal{D}}=\sum_{i}\left(\left(1-\sum_{j} y_{d_{i}, r_{j}}\right) c\left(d_{i}^{t}, d_{i}^{r}\right)\right) \text {. }
$$

Since different cellular users occupy different resource blocks, there is no interference among cellular users. For link $(u, B S)$, however, the interferences from $\mathrm{D} 2 \mathrm{D}$ users and relays can be expressed respectively as

$$
\begin{aligned}
& I_{(u, B S)}^{\mathcal{D}}=\sum_{i} x_{d_{i}, u_{m}}\left(\widetilde{P}_{T}+20 \lg \left(\frac{\lambda}{4 \pi l_{\left(d_{i}^{t}, B S\right)}}\right)\right), \\
& I_{(u, B S)}^{\mathcal{R}}=\sum_{i} x_{r_{i}, u_{m}}\left(\widetilde{P}_{T}+20 \lg \left(\frac{\lambda}{4 \pi l_{\left(r_{i}^{t}, B S\right)}}\right)\right) .
\end{aligned}
$$

The capacity of link $(u, B S)$ is therefore given by

$$
c(u, B S)=\log _{2}\left(\frac{\widetilde{P}_{T}+20 \lg \left(\frac{\lambda}{4 \pi l_{(u, B S)}}\right)}{I_{(u, B S)}^{\mathcal{D}}+I_{(u, B S)}^{\mathcal{R}}+N}+1\right),
$$

and the sum capacity of all cellular users can be expressed as

$$
c_{\mathcal{U}}=\sum_{u} c(u, B S)
$$

The sum capacity $C_{\text {sum }}$ of the network, involving all relay assisted D2D users, ordinary D2D users and cellular users, can therefore be obtained as

$$
c_{\text {sum }}\left(\boldsymbol{X}_{\mathcal{D}}, \boldsymbol{X}_{\mathcal{R}}, \boldsymbol{Y}\right)=c_{\mathcal{R} \mathcal{A}}+c_{\mathcal{D}}+c_{\mathcal{U}} .
$$




\subsection{Relay Selection \& Resource Allocation Problem}

The joint optimization problem of relay selection and resource allocation can be formulated as the one that maximizes the sum capacity $c_{\text {sum }}\left(\boldsymbol{X}_{\mathcal{D}}, \boldsymbol{X}_{\mathcal{R}}, \boldsymbol{Y}\right)$ with the decision variables $\boldsymbol{Y}$ and $\boldsymbol{X}_{\mathcal{D}}, \boldsymbol{X}_{\mathcal{R}}$, subject to certain system constrains.

A D2D pair can only be assisted by one relay or transmit through direct link as an ordinary D2D pair, while a relay either assists two D2D pairs to form a coding region or does not take part in any D2D pair's transmissions. Therefore, the constrains for relay selection are specified by

$$
\begin{aligned}
& y_{d_{i}, r_{j}} \in\{0,1\} \quad \forall i, j, \\
& \sum_{i} y_{d_{i}, r_{j}} \leq 1 \quad \forall j, \\
& \sum_{j} y_{d_{i}, r_{j}} \in\{0,2\} \quad \forall i .
\end{aligned}
$$

For resource allocation, each D2D pair or relay is only allowed to share the spectrum of a single cellular user. In addition, the relay is not allowed to share the same spectrum resource with the D2D pairs it assisted due to half duplex assumption. In the senario of Fig. 2, for example, $r$ will not be able to transmit to $d_{1}^{r}$ and $d_{2}^{r}$ as well as to receive from $d_{1}^{t}$ and $d_{2}^{t}$ at the same time if it occupies the same spectrum resource as $d_{1}^{t}$ or $d_{2}^{t}$. The two relay assisted D2D pairs in a coding region are allowed to share the same spectrum resource. The constrains for resource allocation are thus given by

$$
\begin{aligned}
& x_{d_{i}, u_{k}} \in\{0,1\} \quad \forall i, k, \\
& x_{r_{j}, u_{k}} \in\{0,1\} \quad \forall j, k, \\
& \sum_{i} x_{d_{i}, u_{k}}=1 \quad \forall k, \\
& \sum_{j} x_{r_{j}, u_{k}}=1 \quad \forall k, \\
& x_{d_{i}, u_{k}} x_{r_{j}, u_{k}} y_{d_{i}, r_{j}}=0 \quad \forall i, j, k .
\end{aligned}
$$

Therefore, the optimal relay selection and resource allocation is formulated as the following optimization problem:

$$
\begin{aligned}
& \max c_{\text {sum }}\left(\boldsymbol{X}_{\mathcal{D}}, \boldsymbol{X}_{\mathcal{R}}, \boldsymbol{Y}\right), \\
& \text { s.t. constraints }(39) \text { to }(46) \text { hold. }
\end{aligned}
$$

The above problem is NP-hard. Specifically, it is a nonlinear 0-1 programming problem [24]. The optimization objective (38) also has no obvious convex or concave properties with the decision variables $\boldsymbol{Y}, \boldsymbol{X}_{\mathcal{D}}$ and $\boldsymbol{X}_{\mathcal{R}}$, and we cannot derive the optimal solution by gradient descent. The problem can only be solved by exhaustive search, which takes extremely long time. Moreover, we hope to achieve de-centralized control in our system while still maintaining high performances. This motives us to introduce a two-level de-centralized optimization approach from the view point of game theory, termed NCD2D, which employs coalition formation game and greedy algorithm to solve the problem efficiently.

\section{NC-D2D OVERVIEW}

Since relay selection and resource allocation are closely coupled, the optimal solutions for the two problems must be solved jointly, as in the joint optimization (47). This is because changing the solution of relay selection or resource allocation

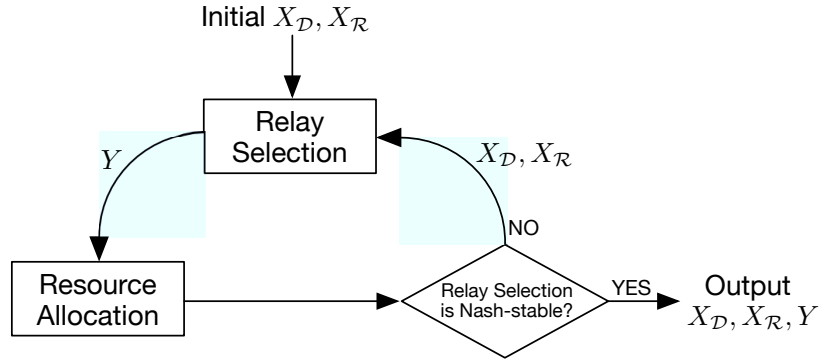

Fig. 4. Illustration of two-level optimization approach of NCD2D.

also changes the solution of the other problem. For example, if two ordinary D2D pairs switch to relay assisted mode and form a coding region with a relay, that is, the solution of relay selection changes. In this case, even though the users and relays remain at the same locations, the interference from other users and relays actually changes. Therefore, once the solution for relay selection $Y$ changes, the solution for resource allocation, $\boldsymbol{X}_{\mathcal{D}}$ and $\boldsymbol{X}_{\mathcal{R}}$, need to be altered accordingly. Resource allocation impacts relay selection in a similar way.

In order to solve the relay selection and resource allocation problems jointly while maintaining low computation complexity, our NC-D2D utilizes a de-centralized two-level optimization approach, where relay selection and resource allocation take place alternately. Fig. 4 shows the operations of NC-D2D. Solving the relay selection problem to obtain $\boldsymbol{Y}$ requires the solutions of resource allocation $\boldsymbol{X}_{\mathcal{D}}$ and $\boldsymbol{X}_{\mathcal{R}}$ as inputs, because the link capacities cannot be calculated without allocating spectrum resources to users and relays. Similarly, optimizing $\boldsymbol{X}_{\mathcal{D}}$ and $\boldsymbol{X}_{\mathcal{R}}$ also requires $\boldsymbol{Y}$ as an input. The operations of NC-D2D can be summarized as follows:

(a) Resource allocation matrices $\boldsymbol{X}_{\mathcal{D}}$ and $\boldsymbol{X}_{\mathcal{R}}$ are randomly generated under the constrains (42) to (45) to serve as the initial input values for relay selection.

(b) Given $\boldsymbol{X}_{\mathcal{D}}$ and $\boldsymbol{X}_{\mathcal{R}}$, the relay selection is solved by a coalition formation game, where each coding region consisting a relay and two related $\mathrm{D} 2 \mathrm{D}$ pairs is a coalition, while all the ordinary D2D pairs form a coalition. D2D pairs swap coalition according a pre-defined preference metric. The coalition formation game continues until the coalition partition reaches Nash-stable state, and it outputs the relay selection matrix $\boldsymbol{Y}$. (c) Given $\boldsymbol{Y}$, the resource allocation is solved with a greedy algorithm based game. All the D2D users and relays take turn to choose a cellular user to share its uplink spectrum resource that achieves highest transmitting capacity, until the process converges to Nash-stable state, with the outputs $\boldsymbol{X}_{\mathcal{D}}$ and $\boldsymbol{X}_{\mathcal{R}}$.

(d) NC-D2D checks if the relay selection's coalition partition is also Nash-stable at this point. If it is, NC-D2D outputs the current $\boldsymbol{Y}, \boldsymbol{X}_{\mathcal{D}}$ and $\boldsymbol{X}_{\mathcal{R}}$ as the joint solution, since the whole system has reached stable state. Otherwise, if the relay selection is no longer stable due to resource allocation, NCD2D repeats steps $(b),(c)$ and $(d)$, until both the relay selection and resource allocation converge to Nash-stable state.

Each time NC-D2D execute steps $(b)$ and $(c)$ is called one round. Our NC-D2D achieves de-centralized control, where each D2D pair and relay only requires local information of the network. This reduces the overhead significantly compared to centralized control, where all the nodes need to be updated 
with the information and topology of the whole network. These two games and their definitions of Nash-stability will be introduced in detail in Sections 5 and 6, respectively.

\section{Relay Selection Coalition formation GAME}

In a coalition game, the players form coalitions to improve the system utility. Since there are two kinds of D2D pairs in our system, relay assisted D2D pairs and ordinary D2D pairs, we consider two kinds of coalitions in the coalition formation game. The first kind of coalition is formed by relay and corresponding relay assisted D2D pairs. Let $\mathcal{F}_{r_{j}}$ represent the coalition of the coding region where $r_{j}$ is in, which also consists of D2D pairs $d_{\alpha(j)}$ and $d_{\beta(j)}$. The number of firstkind coalitions equals to the number of relays in the network, which is fixed. The second kind of coalition is denoted by $\mathcal{F}_{\mathcal{D}}$, which consists of all the ordinary D2D pairs in the network.

In this coalition formation game, the players, namely, D2D pairs, swap coalitions in order to optimize the overall system performance. The decision of whether to swap coalition or not should be made according to a pre-defined preference order that applies to all the players. For the sake of achieving high sum capacity, the metric that defines the preference order in our coalition formation game should be related to the system sum capacity, while each node should be able to obtain it by relying only on local network information.

Two D2D pairs in different coalitions may swap coalitions if the system metric $c_{\text {sum }}\left(\boldsymbol{X}_{\mathcal{D}}, \boldsymbol{X}_{\mathcal{R}}, \boldsymbol{Y}\right)$ can be improved. Since there are two kinds of D2D pairs as well as two kinds of coalitions in the system, there are two kinds of coalition swapping among D2D pairs: swapping among ordinary D2D pairs and relay assisted D2D pairs, as well as swapping among relay assisted D2D pairs.

\subsection{Swapping Among Ordinary D2D Pairs and Relay Assisted D2D Pairs}

Given an initialized coalition partition, for D2D pair $d_{i}$ in $F_{r_{j}}$ and $d_{k}$ in $F_{D}$, if the system sum capacity can be increased after $d_{i}$ and $d_{k}$ swap their coalitions, then $d_{i}$ should leave the coding region of $r_{j}$ to switch to ordinary D2D mode while $d_{k}$ should switch to relay assisted mode and form coding region with $r_{j}$. The system sum capacity defines the preference orders of the players in terms of swapping coalition. It should be noted that we do not need to compute the system sum capacity when trying to determining the preference order. For fixed resource allocation matrices $\boldsymbol{X}_{\mathcal{D}}$ and $\boldsymbol{X}_{\mathcal{R}}$, such a coalition swapping does not change the transmission capacities of other links and coding regions except the capacities of $F_{r_{j}}$ and $d_{k}$, due to the fact that the interferences to the rest of the links remains the same. Therefore, $d_{i}$ and $d_{k}$ should swap coalition if

$$
\begin{aligned}
& c_{\mathcal{C} \mathcal{R}}\left(r_{j}, d_{k}, d_{m}\right)+c\left(d_{i}^{t}, d_{i}^{r}\right) \geq c_{\mathcal{C} \mathcal{R}}\left(r_{j}, d_{i}, d_{m}\right)+c\left(d_{k}^{t}, d_{k}^{r}\right) \\
& \text { and } x_{d_{k}, u} x_{r_{i}, u} y_{d_{k}, r_{i}}=0, \forall u,
\end{aligned}
$$

where $d_{m}$ is the other D2D pair assisted by $r_{j}$. Sinc the system constraints must be maintained, the swapping of coalitions cannot take place if any of the constraints is violated. It can be seen that this swapping process is completely decentralized and only local network information is required by the users.
Specifically, $d_{i}$ only needs to know the capacities of its relay assisted coding region and its direct link, while $d_{k}$ only need to know the capacities of its direct link and the potential coding region involving relay $r_{j}$ and D2D pair $d_{m}$.

In the case that the condition (48) is not satisfied, which means that the two D2D pairs prefer to stay in their current coalitions, a chance for swapping should also be considered. For this chance, we design an acceptance probability for $d_{i}$ and $d_{k}$ to swap coalitions, which is specified as

$$
\begin{aligned}
& \phi_{\mathcal{F}_{\mathcal{D}}, \mathcal{F}_{r_{j}}}\left(T_{N}\right)= \\
& \left\{\begin{array}{l}
\exp \left(\frac{c_{\mathcal{C R}}\left(r_{j}, d_{k}, d_{m}\right)+c\left(d_{i}^{t}, d_{i}^{r}\right)-c_{\mathcal{C R}}\left(r_{j}, d_{i}, d_{m}\right)-c\left(d_{k}^{t}, d_{k}^{r}\right)}{T_{N}}\right), \\
\quad \text { if } x_{d_{k}, u} x_{r_{j}, u} y_{d_{k}, r_{j}}=0, \forall u, \\
0, \quad \text { otherwise, }
\end{array}\right.
\end{aligned}
$$

where $T_{N}=T_{0} / \log (N-1)$ with $T_{0}$ a constant and $N$ the current iteration index. The reason for allowing users to swap coalitions by chance is that the coalition formation based on maximizing the sum capacity is guaranteed to converge to a local optimal solution, which may deviate from the global optimal solution, since the optimization problem is non-convex. This swapping coalitions by chance provides a mechanism for the system to escape from local optimal solutions. The acceptance probability gradually approaches to zero as the number of iterations increases, which ensures that the system is able to form stable coalitions.

\subsection{Swapping Among Relay Assisted D2D Pairs}

For relay assisted D2D pairs $d_{i}$ in coalition $\mathcal{F}_{r_{j}}$ and $d_{k}$ in $\mathcal{F}_{r_{l}}$, where $j \neq l$, they swap their coalitions and form coding regions with the other relays $d_{m}$ and $d_{n}$ if doing so increases the system sum capacity, where $d_{m}$ and $d_{n}$ are the other two D2D pairs in coalitions $\mathcal{F}_{r_{j}}$ and $\mathcal{F}_{r_{l}}$, respectively. More specifically, $d_{i}$ and $d_{k}$ will leave their current coalitions and join $\mathcal{F}_{r_{l}}$ and $\mathcal{F}_{r_{j}}$, respectively, if

$$
\begin{aligned}
& c_{\mathcal{C} \mathcal{R}}\left(r_{j}, d_{k}, d_{m}\right)+c_{\mathcal{C R}}\left(r_{l}, d_{i}, d_{n}\right) \geq c_{\mathcal{C} \mathcal{R}}\left(r_{j}, d_{i}, d_{m}\right) \\
& +c_{\mathcal{C} \mathcal{R}}\left(r_{l}, d_{k}, d_{n}\right), \text { and } x_{d_{k}, u} x_{r_{j}, u} y_{d_{k}, r_{j}}=0, \forall u, \\
& \text { and } x_{d_{i}, u} x_{r_{l}, u} y_{d_{i}, r_{l}}=0, \forall u .
\end{aligned}
$$

Similarly, a chance for $d_{i}$ and $d_{k}$ to swap coalitions should be considered even if the new coalitions are not preferred in terms of the system metric. The acceptance probability is given by

$\phi_{\mathcal{F}_{r_{l}}, \mathcal{F}_{r_{j}}}\left(T_{N}\right)=$

$\left\{\begin{array}{l}\exp \left(\frac{c_{\mathcal{C R}}\left(r_{j}, d_{k}, d_{m}\right)+c_{\mathcal{C R}}\left(r_{l}, d_{i}, d_{n}\right)-c_{\mathcal{C R}}\left(r_{j}, d_{i}, d_{m}\right)-c_{\mathcal{C R}}\left(r_{l}, d_{k}, d_{n}\right)}{T_{N}}\right), \\ \quad \text { if } x_{d_{k}, u} x_{r_{j}, u} y_{d_{k}, r_{j}}=0, \forall u, \\ 0, \text { otherwise, }\end{array}\right.$

with $T_{N}=T_{0} / \log (N-1)$. Again only local network information is used to decide whether to swap coalitions or not.

\subsection{Nash-Stability of Coalition Formation Game in Relay Selection}

The iterative procedure of coalition swapping ends when the coalition partition converges to a Nash-stable state. The definition of Nash-stability for relay selection is as follows. 


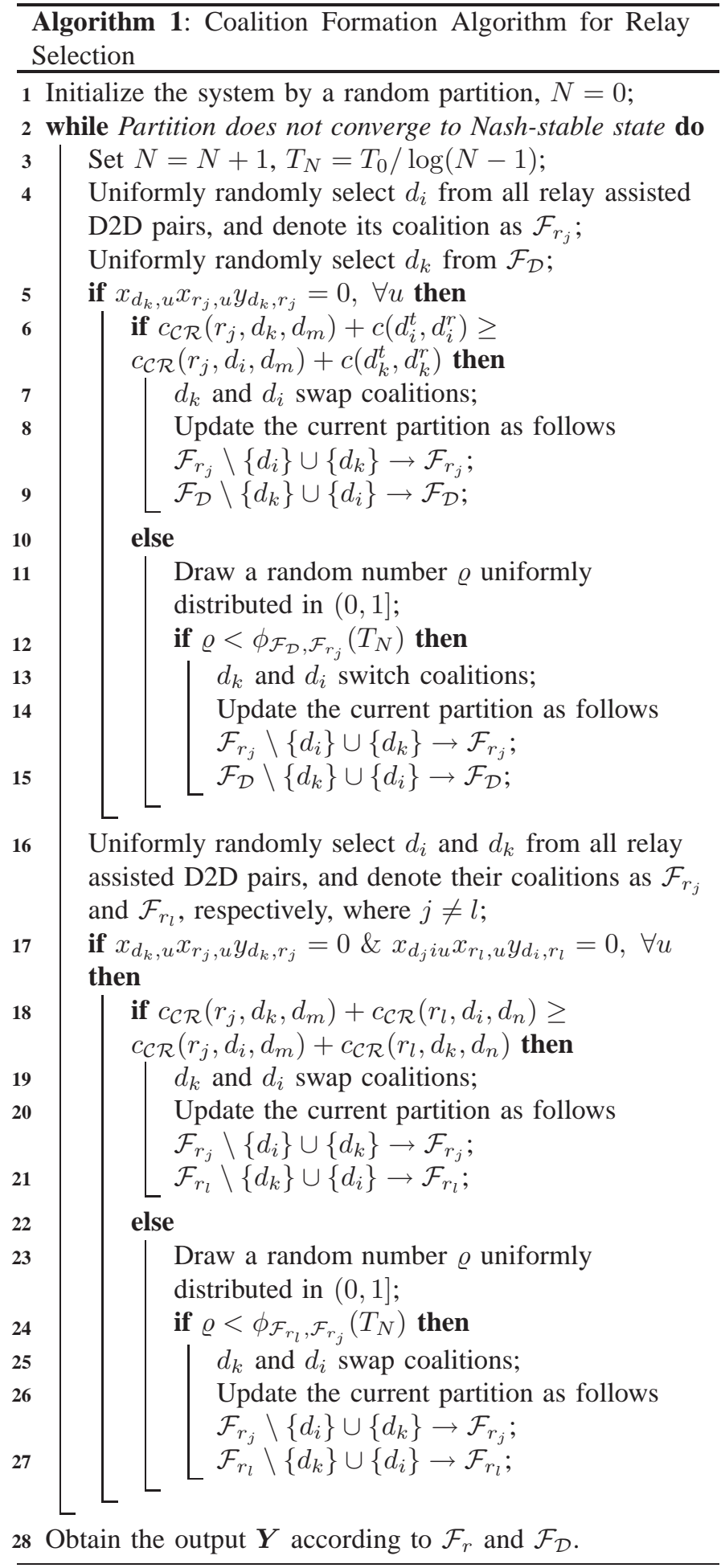

Definition 1: The coalition partition for relay selection is Nash-stable if the sum capacity decreases after $d_{i}$ and $d_{j}$ swap their coalitions for any two D2D pairs $d_{i}$ and $d_{j}$ in the system [25].

Since our optimization problem is non-convex, Nash-stable partition only guarantees a local optimal solution. The complete algorithm of coalition formation game for relay selection is summarized in Algorithm 1.

\subsection{Stability Analysis}

According to the concept from the hedonic games [26], the stability of the final partition depends on weather a Nashstable solution exists for the coalition game. Let us denote the final partition obtained by the coalition game as $\mathcal{F}_{\text {fin }}$. The stability of our proposed game is guaranteed by Theorem 1 .

Theorem 1: Starting from any initial coalition partition, our coalition formation algorithm always converges to Nash-stable partition $\mathcal{F}_{\text {fin }}$ with probability 1 .

Proof: The maximum number of partitions in the game is $R+1$, where $R$ is the number of relays in the network. Therefore, the number of partitions for the D2D pairs set $\mathcal{D}$ is the Bell number in the coalition formation game [27]. Thus, the swapping operations of the D2D pairs will terminate in a finite time with probability 1 , where the system converges to the final stable partition $\mathcal{F}_{\text {fin }}$. Suppose that the final partition $\mathcal{F}_{\text {fin }}$ is not Nash-stable. Then the system capacity will increases if certain D2D pair $d_{i}$ in coalition $\mathcal{F}_{k}$ and $d_{j}$ in $\mathcal{F}_{l}$ swap coalitions, according to the definition of Nashstable. In this case, our algorithm will be able to perform a swap operation for these two D2D pairs with probability 1, which contradicts to the fact that $\mathcal{F}_{\text {fin }}$ is the final partition. This proves that the final coalition partition $\mathcal{F}_{\text {fin }}$ obtained by Algorithm 1 is Nash-stable.

\section{Resource Allocation Game}

Given a relay selection coalition partition $\boldsymbol{Y}$, we use a greedy algorithm based game to solve the resource allocation problem. Similar to relay selection, our resource allocation game has a very low computational complexity and it is capable of converging to a stable local optimal solution.

\subsection{Greedy Algorithm Based Resource Allocation Game}

In the resource allocation game, all the relays and D2D pairs select which spectrum resource to utilize in sequence. To ensure fairness in resource allocation, the sequence is random. Each time a relay or a D2D pair is randomly selected from all the relays and D2D pairs to choose a cellular user to share its spectrum. The selected relay or D2D pair selects the spectrum resource that achieves the highest capacity contribution. If the resource allocation does not converge to Nash-stable state after all the D2D pairs and relays have been allocated with the spectrum resource, a new sequence is generated, according to which each node select the spectrum resource that achieves the highest capacity contribution again. The nodes may select different resources due to the fact that other nodes may occupy different spectrum resources and the interference is different, which leads to variations in link capacities. This process is repeated until the resource allocation is Nash-stable.

The capacity contribution of a node is the contribution of this node to the sum capacity of the network. In selecting spectrum resource, each D2D pair or relay computes its capacity contribution by only utilizing local network information.

Definition 2: For an ordinary D2D pair $d_{i}$, its capacity contribution is the capacity of its direct link $c\left(d_{i}^{t}, d_{i}^{r}\right)$. For a relay assisted D2D pair $d_{i}$ in the coding region of relay $r$ 


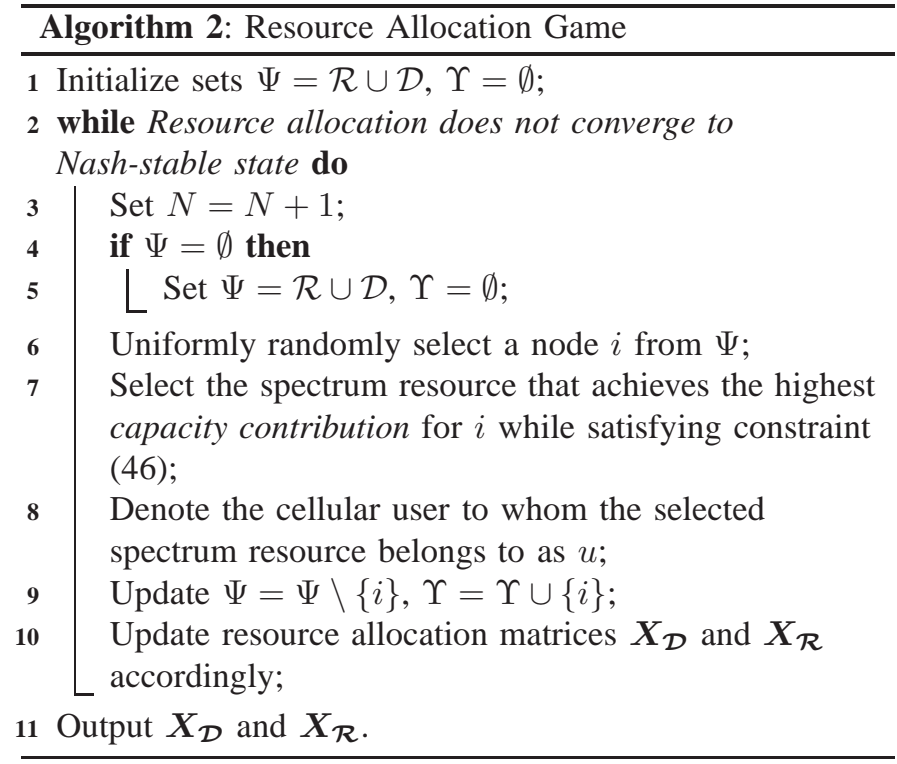

with the other D2D pair $d_{j}$, the capacity contribution of $d_{i}$ is $\min \left\{c\left(d_{i}^{t}, d_{j}^{r}\right), c\left(d_{i}^{t}, r\right)\right\}$. For relay $r$, its capacity contribution is $\min \left\{c\left(r, d_{i}^{r}\right), c\left(r, d_{j}^{r}\right)\right\}$.

Definition 3: The resource allocation $\boldsymbol{X}_{\mathcal{D}}$ and $\boldsymbol{X}_{\mathcal{R}}$ is Nashstable if the capacity contribution of any node $d$ or $r$ decreases after it changes to share a different cellular user's spectrum resource.

The complete algorithm of our resource allocation game is summarized in Algorithm 2.

\subsection{Stability Analysis}

Similar to the relay selection game, the stability of the resource allocation game as given in Algorithm 2 is guaranteed.

Theorem 2: The proposed resource allocation game converges to Nash-stable state with probability 1.

Proof: Although the resource allocation game is not a coalition game, the basic idea of proving its convergences is similar to that for the relay selection game. For each D2D pair or relay, the maximum number of choices for spectrum resource is $C$, which is the number of cellular users. Therefore, the solution space of resource allocation is finite. This guarantees that the convergence probability is 1 . Suppose that the final solution of the resource allocation is not Nashstable. Then there exists some D2D pair $d_{i}$ or relay $r_{j}$ that is able to increase the system capacity by changing to share another cellular user $u_{k}$ 's spectrum resource. Based on our algorithm, the probability of $d_{i}$ or relay $r_{j}$ to select $u_{k}$ 's spectrum resource is 1 , which contradicts to the fact that the current solution is the final stable solution. This proves the Nash-stability of Algorithm 2.

\section{Performance Evaluation}

In this section, we evaluate the performance of our NC-D2D to demonstrate that it is capable of achieving high system sum capacity and outperforming other existing state-of-the-art schemes. We start by introducing our simulation setup. Then the performance of our relay selection coalition formation game and resource allocation game as well as the overall performance of NC-D2D are investigated, respectively.
TABLE 1 Simulation Parameters.

\begin{tabular}{ccc}
\hline Parameter & Symbol & Value \\
\hline Uplink Bandwidth & $W$ & $15 \mathrm{kHz}$ \\
Gaussian noise power & $N$ & $-132 \mathrm{dBm}$ \\
D2D, relay transmission power & $P_{T}^{\mathcal{D}}, P_{T}^{\mathcal{R}}$ & $0 \mathrm{dBm}$ \\
Cellular transmission power & $P_{T}^{\mathcal{U}}$ & $23 \mathrm{dBm}$ \\
User transmitter antenna gain & $G_{T}^{\mathcal{D}}, G_{T}^{\mathcal{R}}, G_{T}^{\mathcal{U}}$ & $0 \mathrm{dBi}$ \\
User receiver antenna gain & $G_{R}^{\mathcal{D}}, G_{R}^{\mathcal{R}}, G_{R}^{\mathcal{U}}$ & $0 \mathrm{dBi}$ \\
BS receiver antenna gain & $G_{R}^{\mathcal{B S}}$ & $14 \mathrm{dBi}$ \\
\hline
\end{tabular}

\subsection{Simulation Setup}

In our simulations, the relays, D2D pairs and cellular users are deployed in a cell, covering a circle area with a radius of $500 \mathrm{~m}$, and the BS is located in the cell center. As mentioned in Section 3, we assume Rayleigh fading, and adopt Friis transmission equation to calculate the path loss of the transmitted signal [23]. We set the uplink bandwidth of each cellular user to be $15 \mathrm{kHz}$. We assume Gaussian noise with the power of $132 \mathrm{dBm}$ for all channels. The transmission power is assumed to be $0 \mathrm{dBm}$ for all relays and $\mathrm{D} 2 \mathrm{D}$ users, and $23 \mathrm{dBm}$ for all cellular users. The antenna gains of all relays, D2D users and cellular users are set to be identical to $0 \mathrm{dBi}$, while the BS's antenna gain is set to be $14 \mathrm{dBi}$ [6]. The parameters of the simulated system are also listed in Table 1. The D2D pairs and cellular users are uniformly randomly distributed in the cell. We simply assume that when two users are within the proximity of $(10,100) \mathrm{m}$, they are able to form a D2D pair. The relays are uniformly randomly deployed on a circle with radius $250 \mathrm{~m}$, centered at the $\mathrm{BS}$.

We evaluate our scheme in five different network setups. The numbers of D2D pairs and cellular users in network setups 1 to 4 are identical, which are 12 and 5 , respectively. The numbers of relays in network setups 1 to 4 are set to $3,4,5$ and 6, respectively. The locations of D2D pairs and cellular users in setups 1 to 4 are identical. That is, we only change the numbers of relays and relays' locations in network setups 1 to 4. The network topology of setup 5 is different from setups 1 to 4 , with 6 relays, 18 D2D pairs, and 8 cellular users.

Since neither the relay selection problem nor the resource allocation problem is convex, NC-D2D only guarantees stable local optimal solutions, which is partially determined by the initial value. Hence we simulate 100 times for each topology and scenario, and evaluate the mean value and the cumulative distribution function (CDF).

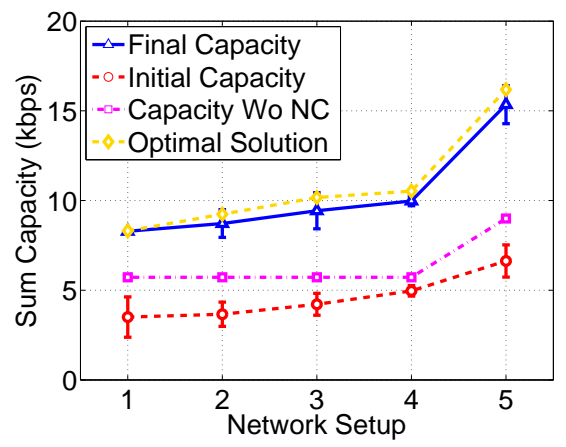

Fig. 5. Comparison of sum capacities in relay selection coalition game under network setups 1 to 5 . 


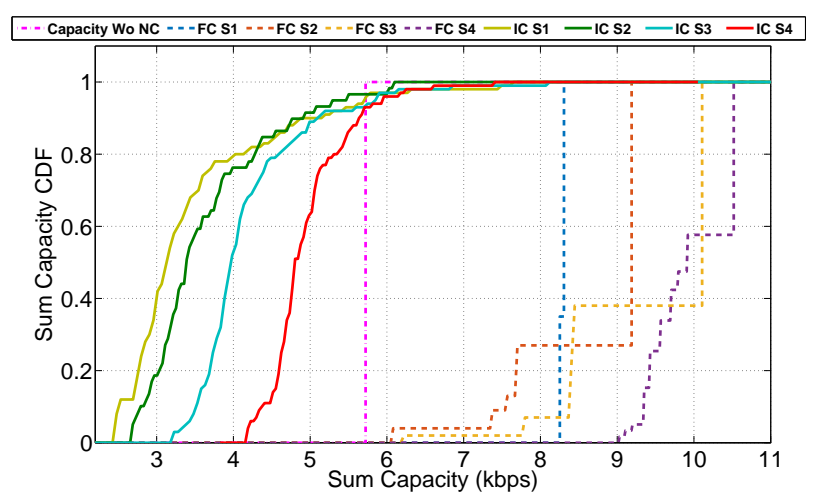

Fig. 6. Comparison of sum-capacity CDFs in relay selection coalition game under network setups 1 to 4 .

\subsection{Relay Selection Coalition Formation Game Per- formance Evaluation}

The mean values and variances of the system sum capacities under network setups 1 to 5 are plotted in Fig. 5, while the CDFs of sum capacity under network setups 1 to 4 are plotted in Fig. 6, and the CDFs of sum capacity under network setup 5 are depicted in Fig. 7. In these figures, 'Capacity Wo NC' indicates the system sum capacity achieved by standard D2D communications without the assistant of either relay nodes or network coding, and 'Final Capacity' or 'FC' indicates the system sum capacity obtained after the relay selection coalition game converges to Nash-stable state. The 'Capacity Wo NC' acts as a reference to indicate the performance gain achievable by relay selection and network coding. In Algorithm 1, the coalition game needs an initial coalition partition, which is randomly generated. The system sum capacity of this randomly generated initial coalition partition is denoted by 'Initial Capacity' or 'IC' in these figures. 'Optimal Solution' in Fig. 5 is the true optimal solution of the relay selection problem, obtained by exhaustive search. Also, labels 'S1' to 'S4' in Fig. 6 represent network setups 1 to 4.

From the results, we observe that the relay selection game increases the sum capacity significantly, compared to the 'Initial Capacity' and the 'Capacity Wo NC', which indicates that the relay selection game helps the system evolving to the local optimal state that achieves higher system capacity. The average capacities after the relay selection game under 5 network setups outperform those of the 'Capacity Wo NC' by $44.83 \%, 52.33 \%, 64.82 \%, 74.28 \%$ and $70.52 \%$, respectively. The results also show that the relay selection game is able

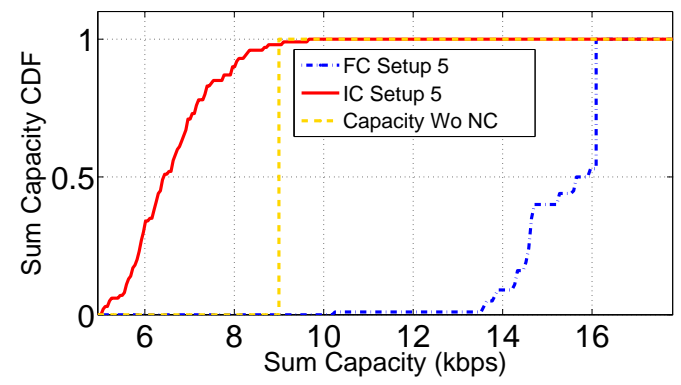

Fig. 7. Comparison of sum-capacity CDFs in relay selection coalition game under network setup 5 . to achieve near-optimal performance. The optimal solutions only outperform our proposed game by $0.23 \%, 5.99 \%, 7.80 \%$, $5.55 \%$, and $5.47 \%$, respectively, under 5 network setups. As expected, the achievable capacity increases with the number of relays, since more relays in the network means that more D2D pairs are able to utilize relay assisted network coding to assist their transmissions. Although our relay selection game only guarantees stable local optimal solutions, we find that it achieves the global optimal solutions in $62 \%, 71 \%, 61 \%$, $42 \%$ and $41 \%$ of all the 100 simulations under network setups 1 to 5 , respectively. The high probability of obtaining the global optimal solution further indicates the effectiveness of our proposed scheme.

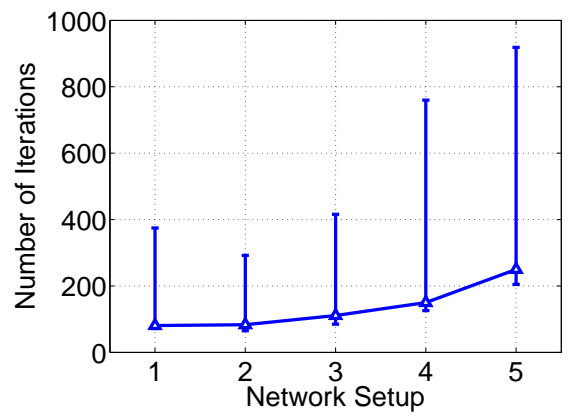

Fig. 8. Number of iterations required by the relay selection coalition game in network setups 1 to 5 .

We also investigate the number of iterations required by the relay selection game to converge to stable solutions in these five setups, and Fig. 8 plots the average values as well as the maximum and minimum values over all the simulations. As expected, the average number of iterations increases with the scale of the network. Most striking observation is that the average numbers of iterations are very close to the minimum values under all the five setups. This indicates that only in very few simulations it costs high number of iterations for the relay selection game to converge, while most of the time the game is able obtain stable solutions very quickly.

\subsection{Resource Allocation Game Performance Evalua- tion}

We next evaluate the performance of the resource allocation game in these five setups. The mean values of the system sum capacities under different network setups are plotted in Fig. 9. It can be seen that our resource allocation game outperforms considerably the random allocation, where each D2D pair and

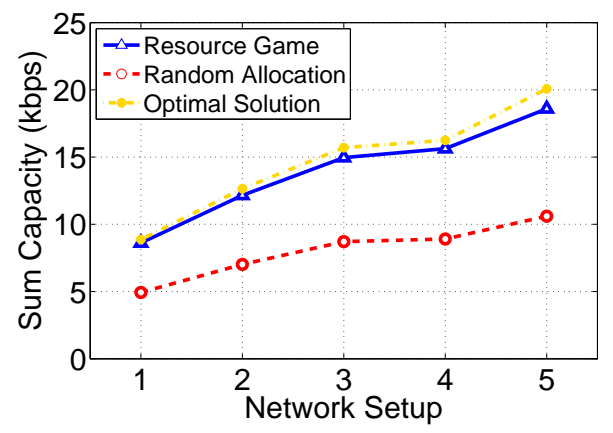

Fig. 9. Comparison of sum capacities in resource allocation game under network setups 1 to 5 . 
relay uniformly and randomly select one cellular user to share its uplink resource. Specifically, the sum capacities achieved by the resource allocation game are $74.79 \%, 73.25 \%, 71.68 \%$, $75.45 \%$ and $75.32 \%$ higher than those of the random allocation under network setups 1 to 5, respectively. Also our resource allocation game attains near-optimal solution, as can be seen from Fig. 9. In particular, the optimal solutions obtained by exhaustive search in these five setups only outperform the sum capacities achieved by our resource allocation game by $1.94 \%$, $2.21 \%, 4.55 \%, 4.29 \%$ and $8.22 \%$, respectively. As expected, the sum capacity achieved by the resource allocation game increases with the network scale.

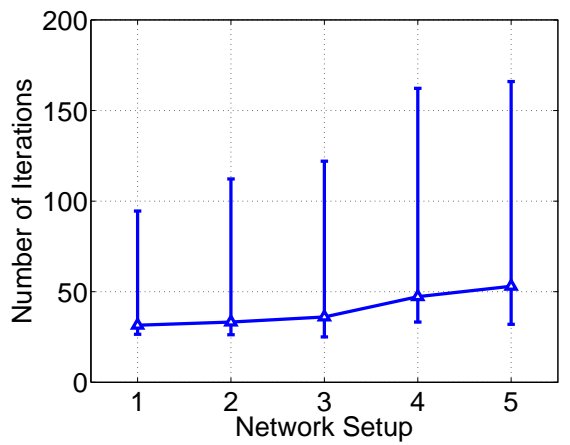

Fig. 10. Number of iterations required by the resource allocation game in network setups 1 to 5 .

Fig. 10 depicts the average number as well as the maximum and minimum numbers of iterations for the resource allocation game to converge. As expected, the number of iterations required increases with the network scale but the maximum numbers of iterations required in all the setups are all fewer then 200. Observe that the average number of iterations is only around 50 for the largest network setup 5. Moreover, the average numbers of iterations are very close to the minimum values under all the five setups. Thus, the resource allocation game converges fast, and it is able to achieve near-optimal solution with very low computation cost.

\subsection{NC-D2D Performance Evaluation}

We now ready to evaluate the performance of the NC-D2D scheme. Fig. 11 plots the CDF of the sum capacity achieved by the NC-D2D under network setup 2, in comparison with those of the relay selection game alone and the resource allocation game alone as well as the sum-capacity CDF obtained without enabling relay assisted network coding, i.e., 'Capcity Wo NC'. Other network setups are omitted since the results are similar to those of Fig. 11. From Fig. 11, it can clearly be seen that

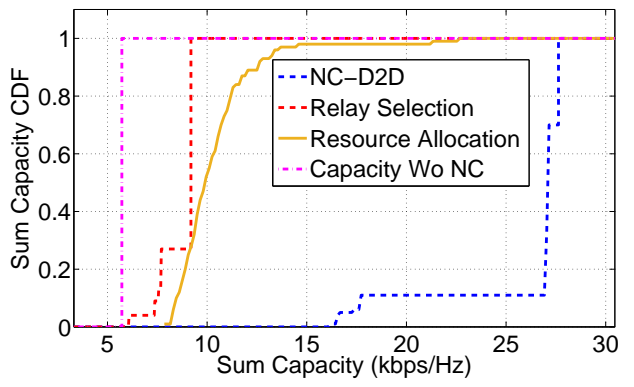

Fig. 11. Sum-capacity CDFs under network setup 2. both the relay selection game alone and the resource allocation game alone outperform the 'Capcity Wo NC', which is just the confirmation of the previous evaluation results. As pointed out previously, the solutions of the relay selection game and the resource allocation game are inter-dependent of each other. By iterating the two games for multiple times until both the relay selection and resource allocation converge to Nash stable solutions in a two-level optimization, the proposed NC-D2D significantly outperforms the results of applying these two games separately. For example, from the CDFs of Fig. 11, we observe that the NC-D2D outperforms the relay selection alone by $135 \%$ in $88 \%$ of the simulations.

To quantify the benefits of this two level-optimization, we define the capacity gain of the NC-D2D in round $i$ as

Capacity gain of round $i=$

$\frac{\text { Capacity of round } i-\text { Capacity of round } i-1}{\text { Capacity of round } i-1}$,

where the Capacity of round 0 is the system sum capacity with a random selection of relays and the associated allocated resources. Obviously, for different network setups and different initial values for the two games, the NC-D2D may take different numbers of rounds to obtain the final stable solution. In all our simulations, the NC-D2D obtains the final stable solutions with no more than three rounds. Fig. 12 depicts the average capacity gain of the NC-D2 in each round. As a common feature of iterative procedure, the capacity increases more slowly as the system converges to the final stable state. Even so, round 3 increases the sum capacity by an average of $40 \%$ over the sum capacity attained in round 2 .

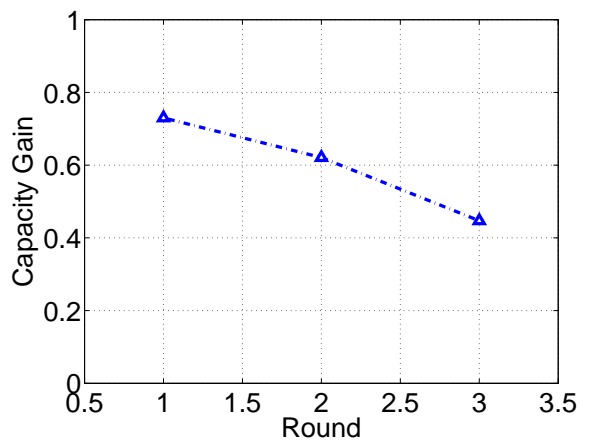

Fig. 12. Average capacity gain of NC-D2D in each round.

Next we plot the average sum capacities and the variances of the NC-D2D in Fig. 13 for network setups 1 to 5, in comparison with the average sum capacities of only applying relay

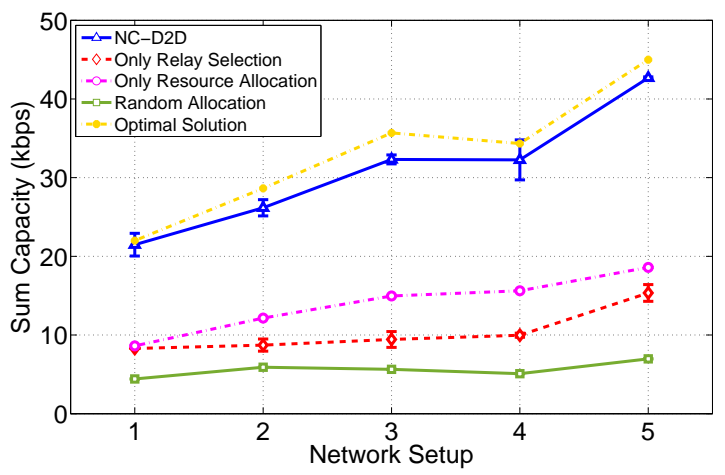

Fig. 13. Sum capacity in network setup 1 to 5 . 
TABLE 2

Average running times by the NC-D2D and the optimal solution.

\begin{tabular}{cccccc}
\hline Algorithm & Setup 1 & Setup 2 & Setup 3 & Setup 4 & Setup 5 \\
\hline NC-D2D & $2.8 \mathrm{~s}$ & $3.1 \mathrm{~s}$ & $3.9 \mathrm{~s}$ & $4.5 \mathrm{~s}$ & $7.3 \mathrm{~s}$ \\
Optimal & $368.6 \mathrm{~s}$ & $416.0 \mathrm{~s}$ & $677.3 \mathrm{~s}$ & $943.5 \mathrm{~s}$ & $2757.1 \mathrm{~s}$ \\
\hline
\end{tabular}

selection, only applying resource allocation, and randomly selecting relays and allocating resources as well as the optimal solution. As expected the NC-D2D outperforms both the relay selection game alone and the resource allocation game alone significantly on all the five setups. Moreover, the performance of the NC-D2D is very closed to those of the optimal solution. Specifically, the optimal solution only outperforms the NCD2D by $2.4 \%, 9.6 \%, 10.2 \%, 6.2 \%$, and $5.3 \%$, respectively, for network setups 1 to 5 .

In the simulation, the optimal solution is obtained by solving the optimization problem (47) based on exhaustive search with pruning. Our simulations were run on Matlab, in a laptop with Mac OS X Yosemite, $2.3 \mathrm{GHz}$ Intel Core i7 processor and 16 GB $1600 \mathrm{MHz}$ DDR3 RAM. Table 2 lists the average times consumed by the NC-D2D and the optimal solution in network setups 1 to 5 . Although the sum capacity achieved by the NCD2D is slightly lower than the optimal solution, the computing time required by the optimal solution is 1000 folds higher that required by the NC-D2D. We also observe that the running time of the NC-D2D scales up linearly with the number of nodes in the network, while the running time of the optimal solution increases exponentially with the network scale. A key reason for the NC-D2D's high efficiency is that it obtains the final solution with only a few rounds. In all our simulations, the NC-D2D converges with no more than three rounds. The percentages of numbers of rounds for the NC-D2D to converge in setups 1 to 5 are plotted in Fig. 14. We can see that about $80 \%$ of the simulations in network setups 1 to 4 , the NC-D2D converges in two rounds, while in setup 5, it converges in two rounds for around $57 \%$ of the simulations.

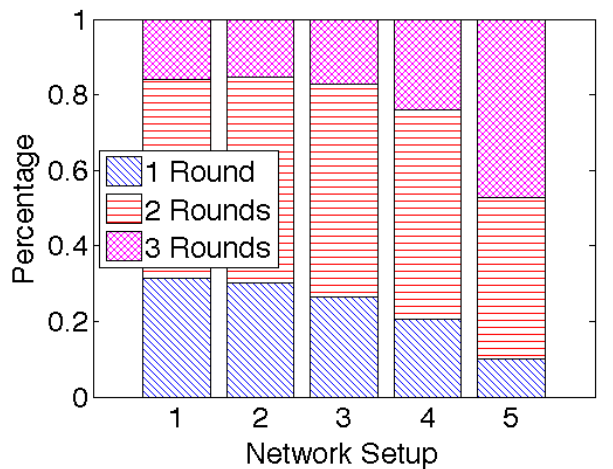

Fig. 14. Percentages of numbers of rounds for the NC-D2D to converge in network setups 1 to 5 .

\section{Conclusions}

In this paper, we have introduced network coding to enhance the performance of D2D communications underlying cellular networks, where one relay aids two D2D pairs' transmissions by performing inter-session network coding. Specifically, we have first formulated the joint problem of relay selection and resource allocation under realistic system constraints for the network coding assisted D2D communications underlying cellular network. Since this optimization is NP-hard, we have proposed a highly efficient near-optimal scheme to solve this joint optimization, referred to as the NC-D2D, which is a twolevel de-centralized optimization scheme that solves the relay selection game and the resource allocation game alternatively. In particular, the relay selection problem is solved with a coalition formation game, where D2D pairs and relays form and swap coalitions for the purpose of enhancing system capacity. The resource allocation game is based on a greedy algorithm to allocate the spectral resource of cellular users to the D2D pairs and relays appropriately. Our extensive simulations have verified that the proposed NC-D2D scheme attains a nearoptimal system sum capacity, while only imposing a fraction of the computational complexity required by the optimal solution. The results thus have demonstrated that the NC-D2D can easily be implemented in practical cellular systems. A future study is to extend the NC-D2D to dynamic networks, where the mobility patterns of users may be utilized.

\section{REFERENCES}

[1] Y. Gu, Y. Zhang, M. Pan, and Z. Han, "Matching and cheating in device to device communications underlying cellular networks," IEEE J. Sel. Areas Commun., vol. 33, no. 10, pp. 2156-2166, Oct. 2015.

[2] X. Lin, J. G. Andrews, A. Ghosh, and R. Ratasuk, "An overview of $3 \mathrm{GPP}$ device-to-device proximity services," IEEE Commun. Mag., vol. 52, no. 4, pp. 40-48, Apr. 2014.

[3] L. Lei, Z. Zhong, C. Lin, and X. Shen, "Operator controlled deviceto-device communications in LTE-advanced networks," IEEE Wireless Commun., vol. 19, no. 3, p. 96-104, Jun. 2012.

[4] Y. Li, D. Jin, J. Yuan, and Z. Han, "Coalitional games for resource allocation in the device-to-device uplink underlaying cellular networks," IEEE Trans. Wireless Commun., vol. 13, no. 7, pp. 3965-3977, Jul. 2014.

[5] C.-H. Yu, K. Doppler, C. B. Ribeiro, and O. Tirkkonen, "Resource sharing optimization for device-to-device communication underlaying cellular networks," IEEE Trans. Wireless Commun., vol. 10, no. 8, pp. 2752-2763, Aug. 2011.

[6] C. Xu, L. Song, Z. Han, Q. Zhao, X. Wang, X. Cheng, and B. Jiao, "Efficiency resource allocation for device-to-device underlay communication systems: a reverse iterative combinatorial auction based approach," IEEE J. Sel. Areas Commun., vol. 31, no. 9, pp. 348-358, Sep. 2013.

[7] N. Lee, X. Lin, J. G. Andrews, and R. W. Heath, "Power control for D2D underlaid cellular networks: modeling, algorithms, and analysis," IEEE J. Sel. Areas Commun., vol. 33, no. 1, pp. 1-13, Jan. 2015.

[8] J. Liu, M. Sheng, X. Wang, Y. Zhang, H. Sun, and J. Li, "Analysis of transmission capacity region in D2D integrated cellular networks with power control," in Proc. ICC 2015 (London), Jun. 8-12, 2015, pp. 2103 2109.

[9] R. Ahlswede, N. Cai, S.-Y. R. Li, and R. W. Yeung, "Network information flow," IEEE Trans. Inform. Theory, vol. 46, no. 4, pp. 1204-1216, Jul. 2000.

[10] P. Pahlevani, M. Hundebøll, M. V. Pedersen, D. E. Lucani, H. Charaf, F. H. Fitzek, H. Bagheri, and M. Katz, "Novel concepts for device-todevice communication using network coding," IEEE Commun. Mag., vol. 52, no. 4, pp. 32-39, Apr. 2014.

[11] T. Ho and H. Viswanathan, "Dynamic algorithms for multicast with intra-session network coding," IEEE Trans. Inform. Theory, vol. 55, no. 2, pp. 797-815, Feb. 2009.

[12] S. Cai, S. Zhang, G. Wu, Y. Dong, and T. Znati, "Minimum cost opportunistic routing with intra-session network coding," in Proc. ICC 2014 (Sydney, NSW), Jun. 10-14, 2014, pp. 502-507.

[13] S. Katti, H. Rahul, W. Hu, D. Katabi, M. Médard, and J. Crowcroft, "XORs in the air: practical wireless network coding," IEEE/ACM Trans. Networking, vol. 16, no. 3, pp. 497-510, Jun. 2008.

[14] Z. Liu, J. Wang, W. Xu, S. Li, and J. Lin, "Inter-session inter-layer network coding-based dual distributed control for heterogeneous-service networks," in Proc. WCNC 2014 (Istanbul, Turkey), Apr. 6-9, 2014, pp. 3230-3235. 
[15] H. Seferoglu, A. Markopoulou, and K. K. Ramakrishnan, "I ${ }^{2} \mathrm{NC}$ : intra- and inter-session network coding for unicast flows in wireless networks," in Proc. INFOCOM 2011 (Shanghai, China), Apr. 10-15, 2011, pp. 1035-1043.

[16] J. Krigslund, J. Hansen, M. Hundeboll, D. E. Lucani, and F. H. Fitzek, "CORE: cope with more in wireless meshed networks," in Proc. VTCSpring 2013 (Dresden, Germany), Jun. 2-5, 2013, pp. 1-6.

[17] A. Bhorkar, C. Ibars, and P. Zong, "Algorithm design and analysis for network coding in D2D underlay communications," in Proc. WCNCW 2015 (New Orleans, LA), Mar. 9-12, 2015, pp. 317-322.

[18] E. M. Maher and K. S. Hassan, "Network coding gain in device-todevice underlaying primary communications," in Proc. CCS 2014 (Rhine River, Germany), Sep. 2-4, 2014, pp. 1-5.

[19] Y. Wu, W. Liu, S. Wang, W. Guo, and X. Chu, "Network coding in device-to-device (D2D) communications underlaying cellular networks," in Proc. ICC 2015 (London), Jun. 8-12, 2015, pp. 2072-2077.

[20] L. Wei, G. Wu, and R. Q. Hu, "Multi-pair device-to-device communications with space-time analog network coding," in Proc. WCNC 2015 (New Orleans, LA), 2015, pp. 920-925.

[21] S. Wen, X. Zhu, Y. Lin, Z. Lin, X. Zhang, and D. Yang, "Achievable transmission capacity of relay-assisted device-to-device (D2D) communication underlay cellular networks," in Proc. VTC Fall-2013 (Las Vegas, NV), Sep. 2-5, 2013, pp. 1-5.

[22] M.-H. Han, B.-G. Kim, and J.-W. Lee, "Subchannel and transmission mode scheduling for D2D communication in OFDMA networks," in Proc. VTC Fall-2012 (Quebec, QC), Sep. 3-6, 2012, pp. 1-5.

[23] H. T. Friis, "A note on a simple transmission formula," Proceedings of the IRE, vol. 34, no. 5, pp. 254-256, May 1946.

[24] T. H. Cormen, C. E. Leiserson, R. L. Rivest, and C. Stein, Introduction to Algorithms. MIT Press, 2001.

[25] A. Bogomolnaia and M. O. Jackson, "The stability of hedonic coalition structures," Games and Economic Behavior, vol. 38, no. 2, pp. 201-230, Feb. 2002.

[26] E. Hossain, D. I. Kim, and V. K. Bhargava, eds., Cooperative Cellular Wireless Networks. Cambridge University Press, 2011.

[27] W. Saad, Z. Han, M. Debbah, A. Hjørungnes, and T. Başar, "Coalitional game theory for communication networks," IEEE Signal Processing Mag., vol. 26, no. 5, pp. 77-97, Sep. 2009. 